\title{
Sustainable recursive social welfare functions
}

\author{
Geir B. Asheim • Tapan Mitra • Bertil Tungodden
}

Received: 11 September 2008 / Accepted: 3 October 2010 / Published online: 26 October 2010 (C) The Author(s) 2010. This article is published with open access at Springerlink.com

\begin{abstract}
What ethical criterion for intergenerational justice should be adopted, e.g., when faced with the task of managing the global environment? Koopmans' axiomatization of discounted utilitarianism is based on seemingly compelling conditions, yet this criterion leads to hard-to-justify outcomes. The present analysis considers a class of sustainable recursive social welfare functions within Koopmans' general framework. This class is axiomatized by means of a weak equity condition ("Hammond Equity for the Future") and general existence is established. Any member of the class
\end{abstract}

\begin{abstract}
We are grateful for helpful discussions with Wolfgang Buchholz, and many constructive comments by anonymous referees, Larry Blume, Walter Bossert, Graciela Chichilnisky, John Hartwick, Aanund Hylland, Larry Karp, Luc Lauwers, Mohamed Mabrouk, Marco Mariotti, Tomoichi Shinotsuka, Yves Sprumont, Kotaro Suzumura, Peter Wakker and seminar participants at Cornell University, Queen's University, Universität Heidelberg, Universität Osnabrück, Université catholique de Louvain and Université de Montréal. Asheim thanks Cornell University and University of California at Santa Barbara for hospitality. This paper is part of the research activities at the center of Equality, Social Organization, and Performance (ESOP) at the Department of Economics at the University of Oslo. ESOP is supported by the Research Council of Norway.
\end{abstract}

\section{G. B. Asheim ( $\varangle)$}

Department of Economics, University of Oslo, Blindern, P.O. Box 1095, 0317 Oslo, Norway e-mail: g.b.asheim@econ.uio.no

\section{T. Mitra}

Department of Economics, Cornell University, 448 Uris Hall, Ithaca, NY 14853, USA

e-mail:tm19@cornell.edu

\section{B. Tungodden}

Department of Economics, Norwegian School of Economics and Business Administration, Helleveien 30, 5045 Bergen, Norway

e-mail: Bertil.Tungodden@nhh.no

B. Tungodden

Chr. Michelsen Institute, P.O. Box 6033, 5892 Bergen, Norway 
satisfies the key axioms of Chichilnisky's “sustainable preferences". The analysis singles out one of Koopmans' original separability conditions (his Postulate 3'a), here called "Independent Present", as particularly questionable from an ethical perspective.

Keywords Intergenerational justice $\cdot$ Sustainability $\cdot$ Discounted utilitarianism

\section{JEL Classification $\quad$ D63 $\cdot$ D71 $\cdot$ Q01}

\section{Introduction}

How should we treat future generations? From a normative point of view, what are the present generation's obligations towards the future? What ethical criterion for intergenerational justice should be adopted if one seeks to respect the interests of future generations? Answering such questions is essential when faced with the task of managing the global environment, e.g., in the context of climate change. ${ }^{1}$

These questions can be approached and answered in at least two ways:

1. Through an axiomatic analysis one can investigate on what ethical conditions various criteria for intergenerational justice are based and then proceed to evaluate the normative appeal of these conditions.

2. By considering different technological environments, one can explore the consequences of various criteria for intergenerational justice, and compare the properties of the intergenerational well-being streams that are generated.

It is consistent with Rawls (1971) reflective equilibrium to do both: criteria for intergenerational justice should be judged both by the ethical conditions on which they build and by their consequences in specific technological environments. In particular, we may question the appropriateness of a criterion for intergenerational justice if it produces unacceptable outcomes in relevant technological environments. This view has been supported by many scholars, including Koopmans (1967), Dasgupta and Heal (1979, p. 311), and Atkinson (2001, p. 206).

When evaluating long-term policies, economists usually suggest to maximize the sum of discounted utilities. On the one hand, such discounted utilitarianism has been given a solid axiomatic foundation by Koopmans (1960). ${ }^{2}$ On the other hand, this criterion has ethically questionable implications when applied to economic models with resource constraints. This is demonstrated by Dasgupta and Heal (1974) in the so-called Dasgupta-Heal-Solow (DHS) model of capital accumulation and resource depletion (Dasgupta and Heal 1974, 1979; Solow 1974), where discounted utilitarianism for any

\footnotetext{
1 A separate set of important questions relates to how to implement policies that are designed to respect the interests of future generations and to assess their effectiveness; see, e.g., in the context of climate change, Burniaux and Martins (2010), Dutta and Radner (2010), Karp and Zhang (2010) and Ostrom (2010). In this context it is also of interest to investigate the validity of the 'Coase theorem', as done by Chipman and Tian (2010). Moreover, as pointed out by Lecocq and Hourcade (2010), optimal policies may require estimates of future intragenerational distribution. Finally, as illustrated by Rezai et al. (2010), in some cases, such policies may benefit all generations, and thus do not represent a question of intergenerational justice.

2 For an alternative set of axioms leading to discounted utilitarianism, see Lauwers (1997).
} 
positive discount rate undermines the well-being of generations in far future, even if sustainable streams with non-decreasing well-being are feasible.

In this paper we revisit Koopmans framework, with numerical representability, sensitivity, and stationarity as its key features. In Sect. 2 we consider conditions that are sufficient to numerically represent the social welfare relation by means of a recursive social welfare function satisfying sensitivity, stationarity, and a condition requiring that the evaluation of two streams with the same present well-being not depend on what that level of well-being is, thereby echoing the analysis of Koopmans (1960, Sects. 3-7). In this framework we introduce an equity condition we call "Hammond Equity for the Future", capturing the following ethical intuition: A sacrifice by the present generation leading to a uniform gain for all future generations cannot lead to a less desirable stream of well-being if the present remains better-off than the future even after the sacrifice. ${ }^{3}$

In Sect. 3 we point out that "Hammond Equity for the Future" is weak, as it is implied by all the standard consequentialist equity conditions suggested in the literature. We show that adding this condition leads to a class of sustainable recursive social welfare functions, where the well-being of the present generation is taken into account if and only if the future is better-off. Furthermore, we establish general existence by means of an algorithmic construction. Finally, we show that any member of this class of sustainable recursive social welfare functions satisfies the key axioms of Chichilnisky (1996) "sustainable preferences", namely "No Dictatorship of the Present" and "No Dictatorship of the Future". 4

In Sect. 4 we offer results that identify which of the conditions used by Koopmans (1960) to axiomatize discounted utilitarianism is particularly questionable from an ethical perspective. The condition in question, referred to as "Independent Present" by us and listed as Postulate $3^{\prime}$ a by Koopmans (1960, Sect. 14), requires that the evaluation of two streams which differ during only the first two periods not depend on what the common continuation stream is. It is only by means of "Independent Present" that Koopmans (1960, Sect. 14) moves beyond the recursive form to arrive at discounted utilitarianism, since this condition allows for additively separable representations when combined with stationarity and the requirement that the evaluation of two streams with the same present well-being not depend on what that level of well-being is (Debreu 1960; Gorman 1968a; Koopmans 1986a).

We suggest in Sect. 4 that "Independent Present"-which in the words of Heal (2005) is "restrictive" and "surely not innocent"-may not be supported by ethical intuition, as it is not obvious that the resolution of a conflict between the first two generations should be independent of how their well-being compares to the well-being of later generations. In our formal analysis, we single out "Independent Present" as the culprit by showing that the addition of this condition contradicts both "Hammond Equity for the Future" and the Chichilnisky (1996) conditions.

In Sect. 5 we apply sustainable recursive social welfare functions for studying optimal harvesting of a renewable resource that yields amenities. In a companion paper

\footnotetext{
3 Our condition is inspired from Hammond (1976) Equity condition, but — as we will see-it is weaker and has not only an egalitarian justification.

4 See Chichilnisky (2010) for an analysis of markets where traders have "sustainable preferences".
} 
(Asheim and Mitra 2010) it is demonstrated how such functions can be used to solve the distributional conflicts in the DHS model. In both settings, our new criterion yields consequences that differ from those of discounted utilitarianism.

Koopmans (1960) has often been interpreted as presenting the definitive case for discounted utilitarianism. In Sect. 6 we discuss how our results contribute to a weakening of this impression, by exploring other avenues within the general setting of his approach. We also investigate the scope for our new equity condition "Hammond Equity for the Future" outside the Koopmans framework by not imposing that the social welfare relation is numerically representable.

All lemmas and proofs are relegated to an Appendix.

\section{Formal setting and basic result}

Let $\mathbb{R}$ denote the set of real numbers and $\mathbb{Z}_{+}$the set of non-negative integers. Denote by ${ }_{0} \mathbf{x}=\left(x_{0}, x_{1}, \ldots, x_{t}, \ldots,\right)$ an infinite stream, where $x_{t} \in Y$ is a one-dimensional indicator of the well-being of generation $t$, and $Y \subseteq \mathbb{R}$ is a non-degenerate interval of admissible well-beings. ${ }^{5}$ We will consider the set $\mathbf{X}$ of infinite streams bounded in well-being (see Koopmans 1986b, p. 89); i.e., $\mathbf{X}$ is given by

$$
\mathbf{X}=\left\{{ }_{0} \mathbf{x} \in \mathbb{R}^{\mathbb{Z}_{+}} \mid\left[\inf _{t} x_{t}, \sup _{t} x_{t}\right] \subseteq Y\right\} .
$$

By setting $Y=[0,1]$, this includes the important special case where $\mathbf{X}=[0,1]^{\mathbb{Z}_{+}}$. However, the formulation allows for cases where $Y$ is not compact.

Denote by ${ }_{0} \mathbf{x}_{T-1}=\left(x_{0}, x_{1}, \ldots, x_{T-1}\right)$ and ${ }_{T} \mathbf{x}=\left(x_{T}, x_{T+1}, \ldots, x_{T+t}, \ldots,\right)$ the $T$-head and the $T$-tail of ${ }_{0} \mathbf{x}$. Write ${ }_{\operatorname{con}} z=(z, z, \ldots)$ for the stream of a constant level of well-being equal to $z \in Y$. Throughout this paper we assume that the indicator of well-being is at least ordinally measurable and level comparable across generations; Blackorby et al. (1984) call this "level-plus comparability".

For all ${ }_{0} \mathbf{x},{ }_{0} \mathbf{y} \in \mathbf{X}$, we write ${ }_{0} \mathbf{x} \geq{ }_{0} \mathbf{y}$ if and only if $x_{t} \geq y_{t}$ for all $t \in \mathbb{Z}_{+},{ }_{0} \mathbf{x}>{ }_{0} \mathbf{y}$ if and only if ${ }_{0} \mathbf{x} \geq 0 \mathbf{y}$ and ${ }_{0} \mathbf{x} \neq{ }_{0} \mathbf{y}$, and ${ }_{0} \mathbf{x} \gg_{0} \mathbf{y}$ if and only if $x_{t}>y_{t}$ for all $t \in \mathbb{Z}_{+}$.

A social welfare relation (SWR) is a binary relation $\succsim$ on $\mathbf{X}$, where for all ${ }_{0} \mathbf{x}, 0 \mathbf{y} \in$ $\mathbf{X},{ }_{0} \mathbf{x} \succsim{ }_{0} \mathbf{y}$ stands for $\left.{ }_{0} \mathbf{x},{ }_{0} \mathbf{y}\right) \in \succsim$ and entails that ${ }_{0} \mathbf{X}$ is deemed socially at least as good as $0 \mathbf{y}$. Denote by $\sim$ and $\succ$ the symmetric and asymmetric parts of $\succsim$; i.e., ${ }_{0} \mathbf{X} \sim{ }_{0} \mathbf{y}$ is equivalent to ${ }_{0} \mathbf{x} \succsim{ }_{0} \mathbf{y}$ and ${ }_{0} \mathbf{y} \succsim{ }_{0} \mathbf{x}$ and entails that ${ }_{0} \mathbf{x}$ is deemed socially indifferent to ${ }_{0} \mathbf{y}$, while ${ }_{0} \mathbf{x} \succ{ }_{0} \mathbf{y}$ is equivalent to ${ }_{0} \mathbf{x} \succsim{ }_{0} \mathbf{y}$ and ${ }_{0} \mathbf{y} \succsim_{0} \mathbf{x}$ and entails that ${ }_{0} \mathbf{x}$ is deemed socially preferred to ${ }_{0} \mathbf{y}$.

All comparisons are made at time 0 . We abuse notation slightly by writing, for $T, T^{\prime} \geq 0,{ }_{T} \mathbf{x}$ and ${ }_{T^{\prime}} \mathbf{y}$ when referring to ${ }_{0} \mathbf{x}^{\prime}$ and ${ }_{0} \mathbf{y}^{\prime}$ where for all $t, x_{t}^{\prime}=x_{T+t}$ and $y_{t}^{\prime}=y_{T^{\prime}+t}$. This notational convention allows us to write ${ }_{T} \mathbf{x}, T^{\prime} \mathbf{y} \in \mathbf{X}$ and ${ }_{T} \mathbf{x} \succsim T^{\prime} \mathbf{y}$.

\footnotetext{
5 A more general framework is, as used by Koopmans (1960), to assume that the well-being of generation $t$ depends on an $n$-dimensional vector $\mathbf{x}_{t}$ that takes on values in a connected set $\mathbf{Y}$. However, by representing the well-being of generation $t$ by a scalar $x_{t}$, we can focus on intergenerational issues. In doing so, we follow, e.g., Diamond (1965), Svensson (1980), Chichilnisky (1996), Basu and Mitra (2003) and Bossert et al. (2007).
} 
It is used throughout the paper, e.g., in the definition of condition IF, in the statement of Lemma 2, and in the proofs of Proposition 2 and Lemma 3.

A social welfare function (SWF) representing $\succsim$ is a mapping $W: \mathbf{X} \rightarrow \mathbb{R}$ with the property that for all ${ }_{0} \mathbf{x},{ }_{0} \mathbf{y} \in \mathbf{X}, W\left(_{0} \mathbf{x}\right) \geq W\left({ }_{0} \mathbf{y}\right)$ if and only if ${ }_{0} \mathbf{x} \succsim{ }_{0} \mathbf{y}$. A mapping $W: \mathbf{X} \rightarrow \mathbb{R}$ is monotone if ${ }_{0} \mathbf{x} \geq 0 \mathbf{y}$ implies $W\left({ }_{0} \mathbf{x}\right) \geq W\left({ }_{0} \mathbf{y}\right)$.

In the present section, we impose conditions on the SWR sufficient to obtain a numerical representation in terms of an SWF with a recursive structure (see Proposition 2 below), similar to, but not identical to Koopmans' (1960, Sects. 3-7).

To obtain a numerical representation, we impose two conditions.

Condition $\mathbf{O}($ Order $) \succsim$ is complete (for all ${ }_{0} \mathbf{x},{ }_{0} \mathbf{y} \in \mathbf{X},{ }_{0} \mathbf{x} \succsim 0 \mathbf{y}$ or $\left.{ }_{0} \mathbf{y} \succsim{ }_{0} \mathbf{x}\right)$ and transitive (for all ${ }_{0} \mathbf{x},{ }_{0} \mathbf{y},{ }_{0} \mathbf{z} \in \mathbf{X},{ }_{0} \mathbf{x} \succsim{ }_{0} \mathbf{y}$ and ${ }_{0} \mathbf{y} \succsim{ }_{0} \mathbf{z}$ imply ${ }_{0} \mathbf{x} \succsim{ }_{0} \mathbf{z}$ ).

Condition RC (Restricted Continuity) For all ${ }_{0} \mathbf{x}, 0 \mathbf{y} \in \mathbf{X}$, if ${ }_{0} \mathbf{x}$ satisfies $x_{t}=z$ for all $t \geq 1$, and the sequence of streams $\left.{ }_{0} \mathbf{x}^{n}\right\rangle_{n \in \mathbb{N}}$ satisfies $\lim _{n \rightarrow \infty} \sup _{t}\left|x_{t}^{n}-x_{t}\right|=0$ with, for each $n \in \mathbb{N},{ }_{0} \mathbf{x}^{n} \in \mathbf{X}$ and ${ }_{0} \mathbf{x}^{n} \nprec{ }_{0} \mathbf{y}$ (resp. ${ }_{0} \mathbf{x}^{n} \nsucc{ }_{0} \mathbf{y}$ ), then ${ }_{0} \mathbf{x} \nprec{ }_{0} \mathbf{y}$ (resp. ${ }_{0} \mathbf{x} \nsucc{ }_{0 \mathbf{y}}$ ).

Condition $\mathbf{R C}$ is weaker than ordinary supnorm continuity as, under condition $\mathbf{R C}$, the stream ${ }_{0} \mathbf{x}$ to which the sequence $\left\langle_{0} \mathbf{x}^{n}\right\rangle_{n \in \mathbb{N}}$ converges is restricted to having a constant level of well-being from period 1 on.

Condition $\mathbf{C}$ (Continuity) For all ${ }_{0} \mathbf{x},{ }_{0} \mathbf{y} \in \mathbf{X}$, if the sequence of streams $\left\langle_{0} \mathbf{x}^{n}\right\rangle_{n \in \mathbb{N}}$ satisfies $\lim _{n \rightarrow \infty} \sup _{t}\left|x_{t}^{n}-x_{t}\right|=0$ with, for each $n \in \mathbb{N},{ }_{0} \mathbf{x}^{n} \in \mathbf{X}$ and $\mathbf{x}^{n} \nprec{ }_{0} \mathbf{y}$ (resp. $\left.{ }_{0} \mathbf{x}^{n} \nsucc{ }_{0} \mathbf{y}\right)$, then ${ }_{0} \mathbf{x} \nprec{ }_{0} \mathbf{y}$ (resp. $\left.{ }_{0} \mathbf{x} \nsucc{ }_{0} \mathbf{y}\right)$.

Condition C is entailed by Koopmans (1960) Postulate 1. As the analysis of Sect. 3 shows, the weaker continuity condition $\mathbf{R C}$ enables us to show existence of sustainable recursive social welfare functions.

The central condition in Koopmans (1960) analysis is the stationarity postulate (Postulate 4). Combined with Koopmans' Postulate $3 \mathrm{~b}$ (the condition requiring that the evaluation of two streams with the same present well-being not depend on what that level of well-being is), the stationarity postulate is equivalent to the following independence condition (where we borrow the name that Fleurbaey and Michel (2003) use for a slightly stronger version of this condition).

Condition IF (Independent Future) For all ${ }_{0} \mathbf{x},{ }_{0} \mathbf{y} \in \mathbf{X}$ with $x_{0}=y_{0},{ }_{0} \mathbf{x} \succsim 0 \mathbf{y}$ if and only if ${ }_{1} \mathbf{x} \succsim 1 \mathbf{y}$.

Condition IF means that an evaluation concerning only generations from the next period on can be made as if the present time (time 0 ) was actually at time 1, i.e., as if generations $\{0,1, \ldots\}$ would have taken the place of generations $\{1,2, \ldots\}$. If we extended our framework to also include comparisons at future times, then IF would imply time consistency as long as the SWR is time invariant.

With the well-being of each generation $t$ expressed by a one-dimensional indicator $x_{t}$, it is uncontroversial to ensure through the following condition that a higher value of $x_{t}$ cannot lead to a socially less preferred stream.

Condition M (Monotonicity) For all ${ }_{0} \mathbf{x},{ }_{0} \mathbf{y} \in \mathbf{X}$, if $_{0} \mathbf{x}>{ }_{0} \mathbf{y}$, then ${ }_{0} \mathbf{y} \nsucc{ }_{0} \mathbf{x}$. 
Combined with the completeness part of condition $\mathbf{O}$, it follows from condition $\mathbf{M}$ that, for all ${ }_{0} \mathbf{x},{ }_{0} \mathbf{y} \in \mathbf{X}$, if $_{0} \mathbf{x} \geq{ }_{0} \mathbf{y}$, then ${ }_{0} \mathbf{x} \succsim{ }_{0} \mathbf{y}$. Condition $\mathbf{M}$ is obviously implied by the "Strong Pareto" condition.

Condition SP (Strong Pareto) For all ${ }_{0} \mathbf{x},{ }_{0} \mathbf{y} \in \mathbf{X}$, if ${ }_{0} \mathbf{x}>{ }_{0} \mathbf{y}$, then ${ }_{0} \mathbf{x} \succ{ }_{0} \mathbf{y}$.

With condition $\mathbf{M}$ we need not impose Koopmans (1960) extreme streams postulate (Postulate 5) and can consider the set of infinite streams bounded in well-being.

As the fifth and final condition of our basic representation result (Proposition 2), we impose the following efficiency condition:

Condition RD (Restricted Dominance) For all $x, z \in Y$, if $x<z$, then $(x, \operatorname{con} z) \prec$ $\operatorname{con} z$.

To evaluate the implications of $\mathbf{R D}$, consider the following three conditions:

Condition WS (Weak Sensitivity) There exist ${ }_{0} \mathbf{x},{ }_{0} \mathbf{y},{ }_{0} \mathbf{z} \in \mathbf{X}$ such that $\left(x_{0},{ }_{1} \mathbf{z}\right) \succ$ $\left(y_{0}, 1 \mathbf{z}\right)$.

Condition DF (Dictatorship of the Future) For all ${ }_{0} \mathbf{x},{ }_{0} \mathbf{y} \in \mathbf{X}$ such that ${ }_{0} \mathbf{x} \succ_{0} \mathbf{y}$, there exist $y, \bar{y} \in Y$, with $y \leq x_{t}, y_{t} \leq \bar{y}$ for all $t \in \mathbb{Z}_{+}$, and $T^{\prime} \in \mathbb{Z}_{+}$such that, for every ${ }_{0} \mathbf{z},{ }_{0} \mathbf{v} \in[y, \bar{y}]^{\mathbb{Z}_{+}},\left({ }_{0} \mathbf{z}_{T-1}, T \mathbf{x}\right) \succ\left({ }_{0} \mathbf{v}_{T-1}, T \mathbf{y}\right)$ for all $T>T^{\prime}$.

Condition NDF (No Dictatorship of the Future) Condition DF does not hold.

Condition SP implies condition RD, which in turn implies condition WS. Condition WS coincides with Koopmans' (1960) Postulate 2. Condition NDF generalizes one of Chichilnisky's (1996) two main axioms to our setting where we consider the set of infinite streams bounded in well-being.

Proposition 1 Assume that the SWR $\succsim$ satisfies conditions $\mathbf{O}$ and IF. Then WS is equivalent to $\mathbf{N D F}$.

As already noted at the end of the introduction, the proof of this and later results are provided in an Appendix.

Since RD strengthens WS, it follows from Proposition 1 that RD ensures "No Dictatorship of the Future", provided that the SWR satisfies conditions $\mathbf{O}$ and IF. To appreciate why we cannot replace RD with an even stronger efficiency condition, we refer to the analysis of Sect. 3 and the impossibility result of Proposition 4.

To state Proposition 2, we introduce the following notation:

$$
\begin{aligned}
\mathcal{U}:= & \{U: Y \rightarrow \mathbb{R} \mid U \text { is continuous and non-decreasing; } \\
& U(Y) \text { is not a singleton }\} \\
\mathcal{U}_{I}:= & \{U: Y \rightarrow \mathbb{R} \mid U \text { is continuous and increasing }\} \\
\mathcal{V}(U):= & \left\{V: U(Y)^{2} \rightarrow \mathbb{R} \mid V \text { satisfies (V.0), (V.1), (V.2), and (V.3) }\right\},
\end{aligned}
$$

where for all $U \in \mathcal{U}, U(Y)$ denotes the range of $U$, and the properties of the aggregator function $V$, (V.0)-(V.3), are as follows:

(V.0) $V(u, w)$ is continuous in $(u, w)$ on $U(Y)^{2}$.

(V.1) $V(u, w)$ is non-decreasing in $u$ for given $w$. 
(V.2) $V(u, w)$ is increasing in $w$ for given $u$.

(V.3) $V(u, w)<w$ for $u<w$, and $V(u, w)=w$ for $u=w$.

Proposition 2 The following two statements are equivalent:

(1) The SWR $\succsim$ satisfies conditions $\mathbf{O}, \mathbf{R C}, \mathbf{I F}, \mathbf{M}$, and $\mathbf{R D}$.

(2) There exists a monotone SWF $W: \mathbf{X} \rightarrow \mathbb{R}$ representing $\succsim$ and satisfying, for some $U \in \mathcal{U}_{I}$ and $V \in \mathcal{V}(U), W\left({ }_{0} \mathbf{x}\right)=V\left(U\left(x_{0}\right), W\left({ }_{1} \mathbf{x}\right)\right)$ for all ${ }_{0} \mathbf{x} \in \mathbf{X}$ and $W(\operatorname{con} z)=U(z)$ for all $z \in Y$.

For a given representation $W$ (with associated utility function $U$ ) of an SWR satisfying conditions $\mathbf{O}, \mathbf{R C}$, IF, $\mathbf{M}$, and $\mathbf{R D}$, we refer to $U\left(x_{t}\right)$ as the utility of generation $t$ and $W\left(_{0} \mathbf{x}\right)$ as the welfare derived from the infinite stream ${ }_{0} \mathbf{x}$.

\section{Hammond equity for the future}

Discounted utilitarianism satisfies conditions $\mathbf{O}, \mathbf{R C}, \mathbf{I F}, \mathbf{M}$, and RD. Hence, these conditions do not by themselves prevent "Dictatorship of the Present", in the terminology of Chichilnisky (1996).

Condition DP (Dictatorship of the Present) For all ${ }_{0} \mathbf{x},{ }_{0} \mathbf{y} \in \mathbf{X}$ such that ${ }_{0} \mathbf{x} \succ{ }_{0} \mathbf{y}$, there exist $\underline{y}, \bar{y} \in Y$, with $\underline{y} \leq x_{t}, y_{t} \leq \bar{y}$ for all $t \in \mathbb{Z}_{+}$, and $T^{\prime} \in \mathbb{Z}_{+}$such that, for any ${ }_{0} \mathbf{z},{ }_{0} \mathbf{v} \in[\underline{y}, \bar{y}]^{\mathbb{Z}_{+}},\left({ }_{0} \mathbf{x}_{T-1},{ }_{T} \mathbf{z}\right) \succ\left({ }_{0} \mathbf{y}_{T-1},{ }_{T} \mathbf{v}\right)$ for all $T>T^{\prime}$.

Condition NDP (No Dictatorship of the Present) Condition DP does not hold.

Condition NDP generalizes the other of Chichilnisky's (1996) two main axioms to our setting where we consider the set of infinite streams bounded in well-being.

We impose a weak new equity condition that ensures NDP. Combined with RC, this condition entails that the interest of the present are taken into account only if the present is worse-off than the future. Consider a stream $(x, \operatorname{con} z)$ having the property that well-being is constant from the second period on. For such a stream we may unequivocally say that, if $x<z$, then the present is worse-off than the future. Likewise, if $x>z$, then the present is better-off than the future.

Condition HEF (Hammond Equity for the Future) For all $x, y, z, v \in Y$, if $x>y>$ $v>z$, then $(x, \operatorname{con} z) \nsucc(y, \operatorname{con} v) .{ }^{6}$

For streams where well-being is constant from the second period on, condition HEF captures the idea of giving priority to an infinite number of future generations in the choice between alternatives where the future is worse-off compared with the present in both alternatives. If the present is better-off than the future and a sacrifice now leads to a uniform gain for all future generations, then such a transfer from the present to the future cannot lead to a less desirable stream, as long as the present remains better-off than the future.

\footnotetext{
6 Condition HEF was introduced in a predecessor to this paper (Asheim and Tungodden 2004b) and has been analyzed by Banerjee (2006), Asheim et al. (2007), Asheim and Mitra (2010), and Alcantud and García-Sanz (2010).
} 
To appreciate the weakness of condition HEF, consider weak versions of the standard "Hammond Equity" condition (Hammond 1976) and Lauwers (1998) nonsubstitution condition.

Condition WHE (Weak Hammond Equity) For all ${ }_{0} \mathbf{x}, 0 \mathbf{y} \in \mathbf{X}$, if ${ }_{0} \mathbf{x}$ and $0 \mathbf{y}$ satisfy that there exists a pair $\tau^{\prime}, \tau^{\prime \prime}$ such that $x_{\tau^{\prime}}>y_{\tau^{\prime}}>y_{\tau^{\prime \prime}}>x_{\tau^{\prime \prime}}$ and $x_{t}=y_{t}$ for all $t \neq \tau^{\prime}, \tau^{\prime \prime}$, then ${ }_{0} \mathbf{x} \nsucc{ }_{0} \mathbf{y}^{7}$

Condition WNS (Weak Non-Substitution) For all $x, y, z, v \in Y$, if $v>z$, then $(x, \operatorname{con} z) \nsucc(y, \operatorname{con} v)$.

By assuming, in addition, that well-beings are at least cardinally measurable and fully comparable, we may also consider weak versions of the Lorenz Domination and Pigou-Dalton principles. Such equity conditions have been used in the setting of infinite streams by, e.g., Birchenhall and Grout (1979), Asheim (1991), Fleurbaey and Michel (2001), and Hara et al. (2008).

Condition WLD (Weak Lorenz Domination) For all ${ }_{0} \mathbf{x},{ }_{0} \mathbf{y} \in \mathbf{X}$, if ${ }_{0} \mathbf{x}$ and ${ }_{0} \mathbf{y}$ are such that ${ }_{0} \mathbf{y}_{T-1}$ weakly Lorenz dominates ${ }_{0} \mathbf{x}_{T-1}$ and ${ }_{T} \mathbf{x}={ }_{T} \mathbf{y}$ for some $T>1$, then ${ }_{0} \mathbf{x} \nsucc{ }_{0} \mathbf{y} .^{8}$

Condition WPD (Weak Pigou-Dalton) For all ${ }_{0} \mathbf{x},{ }_{0} \mathbf{y} \in \mathbf{X}$, if ${ }_{0} \mathbf{x}$ and ${ }_{0} \mathbf{y}$ are such that there exist a positive number $\epsilon$ and a pair $\tau^{\prime}$, $\tau^{\prime \prime}$ satisfying $x_{\tau^{\prime}}-\epsilon=y_{\tau^{\prime}} \geq y_{\tau^{\prime \prime}}=$ $x_{\tau^{\prime \prime}}+\epsilon$ and $x_{t}=y_{t}$ for all $t \neq \tau^{\prime}, \tau^{\prime \prime}$, then ${ }_{0} \mathbf{x} \nsucc{ }_{0} \mathbf{y}$.

While it is clear that condition HEF is implied by WNS, it is perhaps less obvious that, under $\mathbf{O}$ and $\mathbf{M}, \mathbf{H E F}$ is at least as weak as each of WHE, WPD, and WLD.

Proposition 3 Assume that the SWR $\succsim$ satisfies conditions $\mathbf{O}$ and $\mathbf{M}$. Then each of WHE, WPD, and WLD implies HEF.

Note that condition HEF involves a comparison between a sacrifice by a single generation and a uniform gain for each member of an infinite set of generations that are worse-off. Hence, contrary to the standard "Hammond Equity" condition, if wellbeings are made (at least) cardinally measurable and fully comparable, then the transfer from the better-off present to the worse-off future specified in condition HEF increases the sum of well-beings for a sufficiently large number $T$ of generations. This entails that condition HEF is implied by both WPD and WLD, independently of what specific cardinal scale of well-beings is imposed (provided that conditions $\mathbf{O}$ and $\mathbf{M}$ are satisfied). Hence, "Hammond Equity for the Future" can be endorsed from both an egalitarian and utilitarian point of view. In particular, condition HEF is weaker and more compelling than the standard "Hammond Equity" condition.

\footnotetext{
7 Under completeness, condition WHE corresponds to the standard "Hammond Equity" condition, where the premise implies $0 \mathbf{x} \precsim 0 \mathbf{y}$.

${ }^{8}$ For any $T>0,{ }_{0} \mathbf{y}_{T-1}$ weakly Lorenz dominates ${ }_{0} \mathbf{x}_{T-1}$ if and only if (i) $\sum_{\tau=0}^{T-1} y_{\tau}=\sum_{\tau=0}^{T-1} x_{\tau}$ and (ii) if $\varphi$ and $\psi$ are permutations on $\{0, \ldots, T-1\}$ such that $y_{\varphi(1)} \leq \cdots \leq y_{\varphi(T-1)}$ and $x_{\psi(1)} \leq \cdots \leq x_{\psi(T-1)}$, then $\sum_{\tau=0}^{t} y_{\varphi(\tau)} \geq \sum_{\tau=0}^{t} x_{\psi(\tau)}$ for every $t=0, \ldots, T-1$.
} 
However, in line with the Diamond-Yaari impossibility result (Diamond 1965) on the inconsistency of equity and efficiency conditions under continuity, ${ }^{9}$ the equity condition HEF is in conflict with the following weak efficiency condition under RC.

Condition RS (Restricted Sensitivity) There exist $x, z \in Y$ with $x>z$ such that $(x, \operatorname{con} z) \succ \operatorname{con} z$.

Condition SP implies condition RS, which in turn implies condition WS.

\section{Proposition 4 There is no SWR $\succsim$ satisfying conditions $\mathbf{R C}, \mathbf{R S}$, and $\mathbf{H E F}$.}

Impossibility results arising from HEF are further explored in Asheim et al. (2007). Here we concentrate on SwRs that satisfy HEF. We note that it follows from Proposition 4 that RD is the strongest efficiency condition compatible with HEF under RC, when comparing streams $(x, \operatorname{con} z)$ where well-being is constant from the second period on with constant streams $\operatorname{con} z$.

The following result establishes that "Dictatorship of the Present" is indeed ruled out by adding condition HEF to conditions $\mathbf{O}, \mathbf{R C}, \mathbf{I F}$, and $\mathbf{M}$.

Proposition 5 Assume that the SWR $\succsim$ satisfies conditions $\mathbf{O}, \mathbf{R C}, \mathbf{I F}$, and $\mathbf{M}$. Then HEF implies NDP.

How does the basic representation result of Proposition 2 change if we also impose condition HEF on an SWR $\succsim$ satisfying conditions $\mathbf{O}, \mathbf{R C}, \mathbf{I F}, \mathbf{M}$, and RD? To investigate this question, introduce the following notation:

$$
\mathcal{V}_{S}(U):=\left\{V: U(Y)^{2} \rightarrow \mathbb{R} \mid V \text { satisfies (V.0), (V.1), (V.2), and (V.3') }\right\}
$$

where $\left(\mathrm{V} .3^{\prime}\right)$ is given as follows:

$\left(\mathrm{V} .3^{\prime}\right) V(u, w)<w$ for $u<w$, and $V(u, w)=w$ for $u \geq w$.

Note that, for each $U \in \mathcal{U}, \mathcal{V}_{S}(U) \subseteq \mathcal{V}(U)$.

Proposition 6 The following two statements are equivalent:

(1) The SWR $\succsim$ satisfies conditions $\mathbf{O}, \mathbf{R C}, \mathbf{I F}, \mathbf{M}, \mathbf{R D}$, and $\mathbf{H E F}$.

(2) There exists a monotone SWF $W: \mathbf{X} \rightarrow \mathbb{R}$ representing $\succsim$ and satisfying, for some $U \in \mathcal{U}_{I}$ and $V \in \mathcal{V}_{S}(U), W\left({ }_{0} \mathbf{x}\right)=V\left(U\left(x_{0}\right), W\left({ }_{1} \mathbf{x}\right)\right)$ for all ${ }_{0} \mathbf{x} \in \mathbf{X}$ and $W(\operatorname{con} z)=U(z)$ for all $z \in Y$.

We refer to a mapping satisfying the property presented in statement (2) of Proposition 6 as a sustainable recursive SWF. Proposition 6 does not address the question whether there exists a sustainable recursive SWF for any $U \in \mathcal{U}_{I}$ and $V \in \mathcal{V}_{S}(U)$. This question of existence is resolved through the following proposition, which also characterizes the asymptotic properties of such social welfare functions:

\footnotetext{
9 The Diamond-Yaari impossibility result states that the equity condition of "Weak Anonymity" (deeming two streams socially indifferent if one is obtained from the other through a finite permutation of well-beings) is inconsistent with the efficiency condition SP given C. See also Basu and Mitra (2003) and Fleurbaey and Michel (2003).
} 
Proposition 7 For all $U \in \mathcal{U}_{I}$ and $V \in \mathcal{V}_{S}(U)$, there exists a monotone mapping $W$ : $\mathbf{X} \rightarrow \mathbb{R}$ satisfying $W\left({ }_{0} \mathbf{x}\right)=V\left(U\left(x_{0}\right), W\left({ }_{1} \mathbf{x}\right)\right)$ for all ${ }_{0} \mathbf{x} \in \mathbf{X}$ and $W\left({ }_{\operatorname{con}} z\right)=U(z)$ for all $z \in Y$. Any such mapping $W$ satisfies, for each $0 \mathbf{x} \in \mathbf{X}$,

$$
\lim _{T \rightarrow \infty} W\left({ }_{T} \mathbf{x}\right)=\liminf _{t \rightarrow \infty} U\left(x_{t}\right) .
$$

By combining Propositions 6 and 7 we obtain our first main result.

Theorem 1 There exists a class of $\mathrm{SWRs} \succsim$ satisfying conditions $\mathbf{O}, \mathbf{R C}, \mathbf{I F}, \mathbf{M}, \mathbf{R D}$, and HEF.

The proof of the existence part of Proposition 7 is based on an algorithmic construction. For any ${ }_{0} \mathbf{x} \in \mathbf{X}$ and each $T \in \mathbb{Z}_{+}$, consider the following finite sequence:

$$
\left.\begin{array}{l}
w(T, T)=\liminf _{t \rightarrow \infty} U\left(x_{t}\right) \\
w(T-1, T)=V\left(U\left(x_{T-1}\right), w(T, T)\right) \\
\cdots \\
w(0, T)=V\left(U\left(x_{0}\right), w(1, T)\right)
\end{array}\right\}
$$

Define the mapping $W_{\sigma}: \mathbf{X} \rightarrow \mathbb{R}$ by

$$
W_{\sigma}\left({ }_{0} \mathbf{x}\right):=\lim _{T \rightarrow \infty} w(0, T) .
$$

In the proof of Proposition 7 we show that $W_{\sigma}$ is a sustainable recursive SWF.

It is an open question whether $W_{\sigma}$ is the unique sustainable recursive SWF given $U \in \mathcal{U}_{I}$ and $V \in \mathcal{V}_{S}(U)$. As reported in the following proposition, we can show uniqueness if the aggregator function satisfies a condition introduced by Koopmans et al. (1964, p. 88): $V \in \mathcal{V}(U)$ satisfies the property of weak time perspective if there exists a continuous increasing transformation $g: \mathbb{R} \rightarrow \mathbb{R}$ such that $g(w)-g(V(u, w))$ is a non-decreasing function of $w$ for given $u$.

Proposition 8 Let $U \in \mathcal{U}_{I}$ and $V \in \mathcal{V}_{S}(U)$. If $V$ satisfies the property of weak time perspective, then there exists a unique monotone mapping $W: \mathbf{X} \rightarrow \mathbb{R}$ satisfying $W\left({ }_{0} \mathbf{x}\right)=V\left(U\left(x_{0}\right), W\left({ }_{1} \mathbf{x}\right)\right)$ for all $0 \mathbf{x} \in \mathbf{X}$ and $W\left({ }_{\operatorname{con}} z\right)=U(z)$ for all $z \in Y$. This mapping, $W_{\sigma}$, is defined by $(W)$.

We have not been able to establish that the property of weak time perspective follows from the conditions we have imposed. However, it is satisfied in special cases, e.g., with $V$ given by

$$
V(u, w)= \begin{cases}(1-\delta) u+\delta w & \text { if } u<w \\ w & \text { if } u \geq w,\end{cases}
$$

where $\delta \in(0,1) .{ }^{10}$ We can also show that the set of supnorm continuous sustainable recursive SWFs contains at most $W_{\sigma}$. However, even though $W_{\sigma}$ is continuous in the weak sense implied by condition $\mathbf{R C}$, it need not be supnorm continuous.

\footnotetext{
10 Sustainable recursive SWFs with aggregator function given by (2) are analyzed in the companion paper (Asheim and Mitra 2010). Note that an SWR $\succsim$ represented by such a sustainable recursive swF satisfies the following restricted form of the IP condition introduced in the next section:

For all ${ }_{0} \mathbf{x}, 0 \mathbf{y}, 0 \mathbf{z}, 0 \mathbf{v} \in \mathbf{X}$ such that $\left(x_{0}, x_{1}, 2 \mathbf{z}\right),\left(y_{0}, y_{1}, 2 \mathbf{z}\right),\left(x_{0}, x_{1}, 2 \mathbf{v}\right),\left(y_{0}, y_{1}, 2 \mathbf{v}\right)$ are nondecreasing, $\left(x_{0}, x_{1}, 2 \mathbf{z}\right) \succsim\left(y_{0}, y_{1}, 2 \mathbf{z}\right)$ if and only if $\left(x_{0}, x_{1}, 2 \mathbf{v}\right) \succsim\left(y_{0}, y_{1}, 2 \mathbf{v}\right)$.
} 
Once we drop one of the conditions $\mathbf{R C}$, IF, and RD, and combine the remaining two conditions with $\mathbf{O}, \mathbf{M}$, and $\mathbf{H E F}$, new possibilities open up. It is clear that

- The mapping $W: \mathbf{X} \rightarrow \mathbb{R}$ defined by $W\left({ }_{0} \mathbf{x}\right):=\liminf _{t \rightarrow \infty} U\left(x_{t}\right)$ for some $U \in \mathcal{U}_{I}$ represents an SWR satisfying $\mathbf{O}, \mathbf{R C}, \mathbf{I F}, \mathbf{M}$, and $\mathbf{H E F}$, but not RD.

- The maximin SwR satisfies O, RC, M, RD, and HEF, but not IF.

- Leximin and undiscounted utilitarian SWRs for infinite streams satisfy $\mathbf{O}, \mathbf{I F}, \mathbf{M}$, RD, and HEF, but not RC (cf. Proposition 13).

It follows from Propositions 1, 5, and 6 that any sustainable recursive SWF represents an SWR satisfying NDF and NDP. Chichilnisky (1996, Definition 6) defines "sustainable preferences" by imposing NDF and NDP as well as numerical representability and SP. When showing existence in her Theorem 1, she considers SWRs violating condition IF. Hence, through showing general existence for our sustainable recursive SWF, we demonstrate that NDF and NDP can be combined with (a) numerical representability, (b) condition IF which implies stationarity, and (c) sensitivity to present well-being - and thus be imposed within the Koopmans framework-provided that $\mathbf{S P}$ is replaced by weaker dominance conditions. ${ }^{11}$

\section{Independent present}

The following condition is invoked as Postulate 3'a in Koopmans' (1960) characterization of discounted utilitarianism:

Condition IP (Independent Present) For all ${ }_{0} \mathbf{X},{ }_{0} \mathbf{y},{ }_{0} \mathbf{z},{ }_{0} \mathbf{v} \in \mathbf{X},\left(x_{0}, x_{1},{ }_{2} \mathbf{z}\right) \succsim$ $\left(y_{0}, y_{1}, 2 \mathbf{z}\right)$ if and only if $\left(x_{0}, x_{1}, 2 \mathbf{v}\right) \succsim\left(y_{0}, y_{1}, 2 \mathbf{v}\right)$.

Condition IP requires that the evaluation of two streams differing only in the first two periods not depend on what the common continuation stream is. We suggest in this section that this condition may not be compelling, both through appeal to ethical intuition, and through formal results.

We suggest that it might be supported by ethical intuition to accept that the stream $(1,4,5,5,5, \ldots)$ is socially better than $(2,2,5,5,5, \ldots)$, while not accepting that $(1,4,2,2,2, \ldots)$ is socially better than $(2,2,2,2,2, \ldots)$. It is not obvious that we should treat the conflict between the worst-off and the second worst-off generation presented by the first comparison in the same manner as we treat the conflict between the worst-off and the best-off generation put forward by the second comparison.

Turn now to the formal results. Koopmans' (1960) characterizes discounted utilitarianism by means of conditions IF, WS, and IP. However, it turns out that conditions IF, WS, and IP contradict HEF under RC and M. Furthermore, this conclusion is tight, in the sense that an SWR exists if any one of these conditions is dropped. This is our second main result.

\footnotetext{
11 Mitra (2008) shows by means of an example that "sustainable preferences" can be combined with IF in the case where $Y=[0,1]$ if we are willing to give up RC. See also Lauwers (2010) where the constructibility of Chichilnisky (1996) criterion is investigated.
} 
Theorem 2 There is no SWR $\succsim$ satisfying conditions RC, IF, M, WS, HEF, and IP. If one of the conditions $\mathbf{R C}, \mathbf{I F}, \mathbf{M}, \mathbf{W S}, \mathbf{H E F}$, and $\mathbf{I P}$ is dropped, then there exists an $\mathrm{SWR} \succsim$ satisfying the remaining five conditions as well as condition $\mathbf{O}$.

In the following proposition, we reproduce Koopmans' (1960) characterization of discounted utilitarianism within the formal setting of this paper. ${ }^{12}$

Proposition 9 The following two statements are equivalent:

(1) The SWR $\succsim$ satisfies conditions $\mathbf{O}, \mathbf{R C}, \mathbf{I F}, \mathbf{M}, \mathbf{W S}$, and $\mathbf{I P}$.

(2) There exists a monotone SWF $W: \mathbf{X} \rightarrow \mathbb{R}$ representing $\succsim$ and satisfying, for some $U \in \mathcal{U}$ and $\delta \in(0,1), W\left({ }_{0} \mathbf{x}\right)=(1-\delta) U\left(x_{0}\right)+\delta W\left({ }_{1} \mathbf{x}\right)$ for all ${ }_{0} \mathbf{x} \in \mathbf{X}$.

Strengthening $\mathbf{W S}$ to $\mathbf{R D}$ in statement (1) is equivalent to replacing $\mathcal{U}$ by $\mathcal{U}_{I}$ in statement (2).

This proposition follows from standard results for additively separable representations (Debreu 1960; Gorman 1968a; Koopmans 1986a), by exploiting the overlap of periods that conditions IF and IP give rise to (cf. Lemma 3).

Furthermore, we note that the discounted utilitarian SWF exists and is unique.

Proposition 10 For all $U \in \mathcal{U}$ and $\delta \in(0,1)$, there exists a unique monotone mapping $W: \mathbf{X} \rightarrow \mathbb{R}$ satisfying $W\left({ }_{0} \mathbf{x}\right)=(1-\delta) U\left(x_{0}\right)+\delta W\left({ }_{1} \mathbf{x}\right)$ for all ${ }_{0} \mathbf{x} \in \mathbf{X}$. This mapping, $W_{\delta}$, is defined by, for each ${ }_{0} \mathbf{x} \in \mathbf{X}$,

$$
W_{\delta}(0 \mathbf{x})=(1-\delta) \sum_{t=0}^{\infty} \delta^{t} U\left(x_{t}\right) .
$$

Propositions 9 and 10 have the following implication:

Proposition 11 There is no SWR $\succsim$ satisfying conditions $\mathbf{O}, \mathbf{R C}, \mathbf{I F}, \mathbf{M}, \mathbf{I P}, \mathbf{N D P}$, and NDF.

To summarize, it follows from Theorem 2 and Propositions 1 and 11 that, within a Koopmans framework where $\mathbf{O}, \mathbf{R C}, \mathbf{I F}, \mathbf{M}$, and WS are imposed, condition IP contradicts both HEF and NDP. Hence, in such a framework, IP is in conflict with consequentialist equity conditions that respect the interests of future generations.

\section{Applying sustainable recursive SWFs}

We apply sustainable recursive SWFs for studying optimal harvesting of a renewable resource where, following Krautkraemer (1985), well-being may be derived directly from the resource stock. Using discounted utilitarianism in this setting reduces the resource stock below the green golden-rule (defined below) and leads to resource deterioration for sufficiently high discounting (Heal 1998).

\footnotetext{
12 See Bleichrodt et al. (2008) for a simplified characterization of discounted utilitarianism on an extended domain, as well as an overview of related literature.
} 
Maximizing sustainable recursive SWFs leads to very different conclusions, as reported in Proposition 12. Before stating this result, we introduce the model.

The law of motion governing the bio-mass of the renewable resource, $k$, is given by a standard increasing, concave stock-recruitment function, $f$, and therefore the production framework is formally the same as the standard neoclassical aggregate model of economic growth. The function $f: \mathbb{R}_{+} \rightarrow \mathbb{R}_{+}$is assumed to satisfy

(i) $f(0)=0$,

(ii) $f$ is continuous, increasing and strictly concave on $\mathbb{R}_{+}$,

(iii) $\lim _{k \rightarrow 0} \frac{f(k)}{k}>1$ and $\lim _{k \rightarrow \infty} \frac{f(k)}{k}<1$.

It can be shown that there exists a unique number $\bar{k}>0$ such that $f(\bar{k})=\bar{k}$ and $f(k)>k$ for $k \in(0, \bar{k})$.

A feasible path from $k \in[0, \bar{k}]$ is a sequence of resource stocks ${ }_{0} \mathbf{k}$ satisfying

$$
k_{0}=k, \quad 0 \leq k_{t+1} \leq f\left(k_{t}\right) \text { for } t \geq 0 .
$$

It follows from the definition of $\bar{k}$ that $k_{t} \in[0, \bar{k}]$ for $t>0$. Hence, $\bar{k}$ is the maximal attainable resource stock if one starts from an initial stock in $[0, \bar{k}]$. Associated with a feasible path ${ }_{0} \mathbf{k}$ from $k \in[0, \bar{k}]$ is a consumption stream ${ }_{0} \mathbf{c}$, defined by

$$
c_{t}=f\left(k_{t}\right)-k_{t+1} \text { for } t \geq 0 .
$$

Well-being, $x$, depends on consumption and resource amenities through a function $x:[0, \bar{k}]^{2} \rightarrow \mathbb{R}$, which is assumed to satisfy:

(i) $x$ is continuous and quasi-concave on $[0, \bar{k}]^{2}$,

(ii) $x$ is non-decreasing in $(c, k)$, and increasing in $c$ (when $k>0$ ).

The set of admissible well-beings is given by $Y:=[x(0,0), x(\bar{k}, \bar{k})]$. Associated with a feasible path ${ }_{0} \mathbf{k}$ from $k \in[0, \bar{k}]$ is a well-being stream ${ }_{0} \mathbf{x}$, defined by

$$
x_{t}=x\left(f\left(k_{t}\right)-k_{t+1}, k_{t}\right) \text { for } t \geq 0 .
$$

For any $k \in[0, \bar{k}]$, the set of well-being streams associated with feasible resource paths from $k$ is contained in $\mathbf{X}=Y^{\mathbb{Z}_{+}}$.

It follows from the continuity and strict concavity of $f$ and the continuity and quasi-concavity of $x$, combined with property (3)(ii), that there exists a unique number $k^{*} \in[0, \bar{k}]$ such that $x\left(f\left(k^{*}\right)-k^{*}, k^{*}\right) \geq x(f(k)-k, k)$ for all $k \in[0, \bar{k}]$. Since, for any $k \in(0, \bar{k}), x(f(k)-k, k)>x(f(0)-0,0)=x(0,0)$, we have that $k^{*}>0$. Clearly, an additional assumption can be imposed to ensure the existence of $k \in(0, \bar{k})$ such that $x(f(k)-k, k)>x(f(\bar{k})-\bar{k}, \bar{k})=x(0, \bar{k})$, so that $k^{*}<\bar{k}$. The subsequent analysis holds with (and without) any such assumption.

We write $c^{*}:=f\left(k^{*}\right)-k^{*}$ and $x^{*}:=x\left(c^{*}, k^{*}\right)$. By keeping the resource stock constant at $k^{*}$, a maximum sustainable well-being equal to $x^{*}$ is attained; this corresponds to the green golden-rule (Chichilnisky et al. 1995). The following result shows that if $k \in\left[k^{*}, \bar{k}\right]$ and a sustainable recursive SWF is maximized, then welfare corresponds to 
the green golden-rule, and the resource stock never falls below the green golden-rule level.

Proposition 12 Assume that an economy maximizes a sustainable recursive SWF $W$ : $\mathbf{X} \rightarrow \mathbb{R}$ on the set of well-being streams associated with feasible resource paths from $k \in\left[k^{*}, \bar{k}\right]$. Then an optimum exists, and for any optimal resource path $\hat{0} \mathbf{k}$, with associated well-being stream ${ }_{0} \hat{\mathbf{x}}$,

$$
W\left({ }_{t} \hat{\mathbf{x}}\right)=W\left(\operatorname{con} x^{*}\right), \quad \hat{x}_{t} \geq x^{*}, \quad \text { and } \hat{k}_{t} \geq k^{*} \text { for } t \geq 0 .
$$

Hence, in contrast to the existence problem encountered when Chichilnisky's (1996) "sustainable preferences" are applied to such a setting (see Figuieres and Tidball 2010, where this problem motivates an interesting analysis), optima exist when sustainable recursive SWFs are used to evaluate streams (at least, for $k \in\left[k^{*}, \bar{k}\right]$ ). Moreover, in contrast to the outcome under discounted utilitarianism, sustainable recursive SWFs sustain well-being at or above its maximum sustainable level, by sustaining the resource stock at or above the green golden-rule level.

In a companion paper (Asheim and Mitra 2010) it is demonstrated how sustainable recursive SWFs can be used to resolve in an appealing way the interesting distributional conflicts that arise in the DHS model of capital accumulation and resource depletion. In particular, applying sustainable recursive SWFs in this setting leads to growth and development at first when capital is productive, while protecting the generations in the distant future from the grave consequences of discounting when the vanishing resource stock undermines capital productivity.

\section{Concluding remarks}

Koopmans (1960) has often been interpreted as presenting the definitive case for discounted utilitarianism. In Sects. 2 and 3 we have sought to weaken this impression by exploring other avenues within the general setting of his approach. In particular, by not imposing condition IP, used by Koopmans (1960) to characterize discounted utilitarianism, we have been able to combine our new equity condition HEF with the essential features of the Koopmans framework: (a) numerical representability, (b) sensitivity to the interests of the present generation, and (c) condition IF which includes Koopmans' stationarity postulate. This leads to a non-empty class of sustainable recursive social welfare functions. We have argued that condition HEF is weak, as it is implied by all the standard consequentialist equity conditions suggested in the literature, yet strong enough to ensure that the Chichilnisky (1996) conditions are satisfied. As we have discussed in Sect. 5, sustainable recursive social welfare functions are applicable and yield consequences that differ from those of discounted utilitarianism.

In this final section we note that even wider possibilities open up if we are willing to give up numerical representability by not imposing RC. In particular, we are then able to combine the equity condition HEF and the independence condition IP with our basic conditions $\mathbf{O}$ and $\mathbf{I F}$, while strengthening our efficiency conditions $\mathbf{M}$ and $\mathbf{R D}$ to condition $\mathbf{S P}$. 
Proposition 13 There exists an SWR $\succsim$ satisfying conditions $\mathbf{O}$, IF, SP, HEF, and IP.

The proof of this proposition employs the leximin and undiscounted utilitarian SWRs for infinite streams that have been axiomatized in recent contributions (see, Asheim and Tungodden 2004a; Basu and Mitra 2007; Bossert et al. 2007).

We end by making the observation that continuity is not simply a "technical" condition without ethical content. In a setting where $\mathbf{R C}$ (or a stronger continuity condition like $\mathbf{C}$ ) is combined with $\mathbf{R S}$ (or a stronger efficiency condition like SP), it follows from Proposition 4 that condition $\mathbf{H E F}$ is not satisfied. Hence, on this basis one may claim that, in combination with a sufficiently strong efficiency condition, continuity rules out SWFs that protect the interests of future generations by implying that the equity condition HEF does not hold. In the main analysis of this paper we have avoided the trade-off between continuity and numerical representability on the one hand, and the ability to impose the equity condition HEF on the other hand, by weakening the efficiency condition in an appropriate way.

\section{Appendix: Proofs}

Proof of Proposition 1 Part I: WS implies NDF. Assume that the SWR $\succsim$ satisfies conditions $\mathbf{O}$ and WS. By WS, there exist ${ }_{0} \mathbf{x},{ }_{0} \mathbf{y} \in \mathbf{X}$ with ${ }_{1} \mathbf{x}={ }_{1} \mathbf{y}$ such that ${ }_{0} \mathbf{x} \succ_{0} \mathbf{y}$. Let ${ }_{0} \mathbf{z},{ }_{0} \mathbf{v} \in \mathbf{X}$ be given by ${ }_{0} \mathbf{z}={ }_{0} \mathbf{v}={ }_{0} \mathbf{X}$. We have that, for any $\underline{y}, \bar{y} \in Y$ satisfying $y \leq$ $x_{t}, y_{t} \leq \bar{y}$ for all $t \in \mathbb{Z}_{+},{ }_{0} \mathbf{z},{ }_{0} \mathbf{v} \in[\underline{y}, \bar{y}]^{\mathbb{Z}_{+}}$. Still, for all $T>0,\left({ }_{0} \mathbf{z}_{T-1},{ }_{T} \mathbf{x}\right)=_{0} \mathbf{x}=$ $\left({ }_{0} \mathbf{x}_{T-1},{ }_{T} \mathbf{y}\right)=\left({ }_{0} \mathbf{v}_{T-1},{ }_{T} \mathbf{y}\right)$, implying by $\mathbf{O}$ that $\left({ }_{0} \mathbf{z}_{T-1},{ }_{T} \mathbf{x}\right) \sim\left({ }_{0} \mathbf{v}_{T-1},{ }_{T} \mathbf{y}\right)$. This contradicts DF.

Part II: NDF implies WS. Assume that the SWR $\succsim$ satisfies conditions $\mathbf{O}$ and IF. Suppose that WS does not hold, i.e., for all ${ }_{0} \mathbf{x}^{\prime},{ }_{0} \mathbf{y}^{\prime} \in \mathbf{X}$ with ${ }_{1} \mathbf{x}^{\prime}={ }_{1} \mathbf{y}^{\prime},{ }_{0} \mathbf{x}^{\prime} \sim{ }_{0} \mathbf{y}^{\prime}$.

Case (i): There exist ${ }_{0} \mathbf{X},{ }_{0} \mathbf{y} \in \mathbf{X}$ such that ${ }_{0} \mathbf{X} \succ{ }_{0} \mathbf{y}$. Suppose ${ }_{0} \mathbf{x},{ }_{0} \mathbf{y} \in \mathbf{X}$ are such that $_{0} \mathbf{X} \succ{ }_{0} \mathbf{y}$. Let ${ }_{0} \mathbf{z},{ }_{0} \mathbf{v}$ be arbitrary streams in $\mathbf{X}$. We have that ${ }_{T-1} \mathbf{x} \sim\left(z_{T-1},{ }_{T} \mathbf{x}\right)$ for all $T>0$ since WS does not hold. By IF and the above argument,

$$
T-2 \mathbf{x}=\left(x_{T-2}, T-1 \mathbf{x}\right) \sim\left(x_{T-2}, z_{T-1},{ }_{T} \mathbf{x}\right) \sim\left(T_{T-2} \mathbf{z}_{T-1},{ }_{T} \mathbf{x}\right) .
$$

By invoking $\mathbf{O}$ and applying IF and the above argument repeatedly, it follows that ${ }_{0} \mathbf{x} \sim\left({ }_{0} \mathbf{z}_{T-1},{ }_{T} \mathbf{x}\right)$ for all $T>0$. Likewise, ${ }_{0} \mathbf{y} \sim\left({ }_{0} \mathbf{v}_{T-1}, T \mathbf{y}\right)$ for all $T>0$. By $\mathbf{O}$, $\left({ }_{0} \mathbf{z}_{T-1}, T_{T} \mathbf{x}\right) \succ\left({ }_{0} \mathbf{v}_{T-1},{ }_{T} \mathbf{y}\right)$ for all $T>0$. This establishes DF, implying that NDF does not hold.

Case (ii): There do not exist ${ }_{0} \mathbf{x},{ }_{0} \mathbf{y} \in \mathbf{X}$ such that ${ }_{0} \mathbf{x} \succ{ }_{0} \mathbf{y}$. Then $\mathbf{D F}$ is true trivially, implying that NDF does not hold in this case either.

The following lemma is useful for proving Proposition 2 and subsequent results:

Lemma 1 Assume that the SWR $\succsim$ satisfies conditions $\mathbf{O}, \mathbf{R C}$, and $\mathbf{M}$. Then, for all ${ }_{0} \mathbf{X} \in \mathbf{X}$, there exists $z \in Y$ such that ${ }_{\text {con }} z \sim{ }_{0} \mathbf{X}$. If condition $\mathbf{R D}$ is added, then $z$ is unique.

Proof Assume that the SwR $\succsim$ satisfies conditions $\mathbf{O}, \mathbf{R C}$, and $\mathbf{M}$. By $\mathbf{O}, \mathbf{M}$, and the definition of $\mathbf{X}$, there exists $z \in Y$ such that inf $\left\{v \in Y \mid \operatorname{con} v \succsim{ }_{0} \mathbf{X}\right\} \leq z \leq \sup \{v \in$ $\left.Y \mid \operatorname{con} v \precsim{ }_{0} \mathbf{X}\right\}$. By $\mathbf{O}$ and $\mathbf{R C}, \operatorname{con} z \sim{ }_{0} \mathbf{X}$. 
If condition $\mathbf{R D}$ is added, then by $\mathbf{O}, \mathbf{M}$, and $\mathbf{R D}$ we have that

$$
\operatorname{con} v=(v, \operatorname{con} v) \precsim(v, \operatorname{con} z) \prec \operatorname{con} z \text { if } v<z,
$$

so that $\inf \{v \in Y \mid \operatorname{con} v \succsim \mathbf{x}\}=\sup \left\{v \in Y \mid \operatorname{con}_{0} v \precsim{ }_{0} \mathbf{x}\right\}$ and $z$ is unique.

Proof of Proposition 2 Part I: (1) implies (2). Assume that the SWR $\succsim$ satisfies conditions $\mathbf{O}, \mathbf{R C}, \mathbf{I F}, \mathbf{M}$, and $\mathbf{R D}$. In view of Lemma 1 , determine $W: \mathbf{X} \rightarrow Y$ by, for all ${ }_{0} \mathbf{x} \in \mathbf{X}, W\left(_{0} \mathbf{x}\right)=z$ where ${ }_{\operatorname{con}} z \sim{ }_{0} \mathbf{x}$. By $\mathbf{O}$ and $(4), W\left({ }_{0} \mathbf{x}\right) \geq W\left({ }_{0} \mathbf{y}\right)$ if and only if $0 \mathbf{x} \succsim 0 \mathbf{y}$. By $\mathbf{M}, W$ is monotone.

Let $U \in \mathcal{U}_{I}$ be given by $U(x)=x$ for all $x \in Y$, implying that $U(Y)=Y$. Hence, by construction of $W, W(\operatorname{con} z)=z=U(z)$ for all $z \in Y$. It follows from IF that, for given $x_{0} \in Y$, there exists an increasing transformation $V\left(U\left(x_{0}\right), \cdot\right): Y \rightarrow Y$ such that, for all ${ }_{1} \mathbf{x} \in \mathbf{X}, W\left(x_{0},{ }_{1} \mathbf{x}\right)=V\left(U\left(x_{0}\right), W\left({ }_{1} \mathbf{x}\right)\right)$. This determines $V: Y \times Y \rightarrow$ $Y$, where $V(u, w)$ is increasing in $w$ for given $u$, establishing that $V$ satisfies (V.2). By $\mathbf{M}, V(u, w)$ is non-decreasing in $u$ for given $w$, establishing that $V$ satisfies (V.1). Since $(x, \operatorname{con} z) \nprec \operatorname{con} v(\operatorname{resp} .(x, \operatorname{con} z) \nsucc \operatorname{con} v)$ if and only if

$$
V(x, z)=V(U(x), W(\operatorname{con} z))=W(x, \operatorname{con} z) \geq v \quad(\text { resp. } \leq v),
$$

RC implies that $V$ satisfies (V.0). Finally, since

$$
\begin{aligned}
& V(z, z)=V(U(z), W(\operatorname{con} z))=W(\operatorname{con} z)=z \\
& V(x, z)=V(U(x), W(\operatorname{con} z))=W(x, \operatorname{con} z)<W(\operatorname{con} z)=z \quad \text { if } x<z
\end{aligned}
$$

by invoking RD, it follows that $V$ satisfies (V.3). Hence, $V \in \mathcal{V}(U)$.

Part II: (2) implies (1). Assume that the monotone mapping $W: \mathbf{X} \rightarrow \mathbb{R}$ is an SWF and satisfies, for some $U \in \mathcal{U}_{I}$ and $V \in \mathcal{V}(U), W\left({ }_{0} \mathbf{x}\right)=V\left(U\left(x_{0}\right), W\left({ }_{1} \mathbf{x}\right)\right)$ for all ${ }_{0} \mathbf{X} \in \mathbf{X}$ and $W(\operatorname{con} z)=U(z)$ for all $z \in Y$. Since the SWR $\succsim$ is represented by the SWF $W$, it follows that $\succsim$ satisfies $\mathbf{O}$. Moreover, $\succsim$ satisfies $\mathbf{M}$ since $W$ is monotone, $\succsim$ satisfies IF since $V$ satisfies (V.2), and $\succsim$ satisfies RD since $U \in \mathcal{U}_{I}$ and $V$ satisfies (V.3). The following argument shows that $\succsim$ satisfies RC:

Let ${ }_{0} \mathbf{X},{ }_{0} \mathbf{y} \in \mathbf{X}$, and let $x_{t}=z$ for all $t \geq 1$. Let ${ }_{0} \mathbf{x}^{n} \in \mathbf{X}$ for $n \in \mathbb{N}$, with the property that $\lim _{n \rightarrow \infty} \sup _{t}\left|x_{t}^{n}-x_{t}\right|=0$ and, for each $n \in \mathbb{N},{ }_{0} \mathbf{x}^{n} \nprec{ }_{0} \mathbf{y}$. We have to show that ${ }_{0} \mathbf{x} \nprec{ }_{0} \mathbf{y}$, or equivalently, $W\left({ }_{0} \mathbf{x}\right) \geq W\left({ }_{0} \mathbf{y}\right)$. Define $\epsilon_{0}(n)$ and $\epsilon(n)$ for $n \in \mathbb{N}$ by, for each $n \in \mathbb{N}, \epsilon_{0}(n):=\max \left\{0, x_{0}^{n}-x_{0}\right\}$ and $\epsilon(n):=\max \left\{0, \sup _{t \geq 1}\left(x_{t}^{n}-x_{t}\right)\right\}$, so that $\lim _{n \rightarrow \infty} \epsilon_{0}(n)=0$ and $\lim _{n \rightarrow \infty} \epsilon(n)=0$. For each $n \in \mathbb{N}$,

$$
\begin{aligned}
V\left(U\left(x_{0}+\epsilon_{0}(n)\right), U(z+\epsilon(n))\right) & =V\left(U\left(x_{0}+\epsilon_{0}(n)\right), W(\operatorname{con}(z+\epsilon(n)))\right) \\
& =W\left(x_{0}+\epsilon_{0}(n), \operatorname{con}(z+\epsilon(n))\right) \\
& \geq W\left({ }_{0} \mathbf{x}^{n}\right) \geq W\left({ }_{0} \mathbf{y}\right)
\end{aligned}
$$

since $W$ is monotone and represents $\succsim$, and ${ }_{0} \mathbf{x}^{n} \nprec{ }_{0} \mathbf{y}$. This implies that

$$
W\left({ }_{0} \mathbf{x}\right)=V\left(U\left(x_{0}\right), W\left({ }_{\operatorname{con}} z\right)\right)=V\left(U\left(x_{0}\right), U(z)\right) \geq W\left({ }_{0} \mathbf{y}\right)
$$


since $U$ and $V$ are continuous and $\lim _{n \rightarrow \infty} \epsilon(n)=0$. The same kind of argument can be used to show that ${ }_{0} \mathbf{x} \nsucc{ }_{0} \mathbf{y}$ if, for each $n \in \mathbb{N},{ }_{0} \mathbf{x}^{n} \nsucc{ }_{0} \mathbf{y}$.

Proof of Proposition 3 Assume $x>y>v>z$. We must show under $\mathbf{O}$ and $\mathbf{M}$ that each of WHE, WLD, and WPD implies $(x, \operatorname{con} z) \nsucc(y, \operatorname{con} v)$.

Since $x>y>v>z$, there exist an integer $T$ and utilities $x^{\prime}, z^{\prime} \in Y$ satisfying $y>x^{\prime} \geq v>z^{\prime}>z$ and $x-x^{\prime}=T\left(z^{\prime}-z\right)$.

By $\mathbf{O}$ (completeness) and WHE, $\left(x^{\prime}, z^{\prime}, \operatorname{con} z\right) \succsim(x, \operatorname{con} z)$, and by $\mathbf{M},(y, \operatorname{con} v) \succsim$ $\left(x^{\prime}, z^{\prime}, \operatorname{con} z\right)$. By $\mathbf{O}$ (transitivity), $(y, \operatorname{con} v) \succsim(x, \operatorname{con} z)$.

Consider next WLD and WPD. Let ${ }_{0} \mathbf{x}^{0}=(x$, con $z)$, and define ${ }_{0} \mathbf{x}^{n}$ for $n \in$ $\{1, \ldots, T\}$ inductively as follows:

$$
\begin{array}{ll}
x_{t}^{n}=x_{t}^{n-1}-\left(z^{\prime}-z\right) & \text { for } t=0 \\
x_{t}^{n}=z^{\prime} & \text { for } t=n \\
x_{t}^{n}=x_{t}^{n-1} & \text { for } t \neq 0, n .
\end{array}
$$

By $\mathbf{O}$ (completeness) and WLD, ${ }_{0} \mathbf{x}^{T} \succsim{ }_{0} \mathbf{x}^{0}$, and by $\mathbf{M},(y, \operatorname{con} v) \succsim{ }_{0} \mathbf{x}^{T}$. By $\mathbf{O}$ (transitivity), $(y, \operatorname{con} v) \succsim(x, \operatorname{con} z)$ since ${ }_{0} \mathbf{x}^{0}=(x, \operatorname{con} z)$.

By $\mathbf{O}$ (completeness) and WPD, ${ }_{0} \mathbf{x}^{n} \succsim{ }_{0} \mathbf{x}^{n-1}$ for $n \in\{1, \ldots, T\}$, and by $\mathbf{M}$, $(y, \operatorname{con} v) \succsim{ }_{0} \mathbf{x}^{T}$. By $\mathbf{O}$ (transitivity), $(y, \operatorname{con} v) \succsim(x, \operatorname{con} z)$ since ${ }_{0} \mathbf{x}^{0}=(x, \operatorname{con} z)$.

Proof of Proposition 4 This follows from Asheim et al. (2007, Proposition 2).

Proof of Proposition 5 Assume that the SwR $\succsim$ satisfies conditions $\mathbf{O}, \mathbf{R C}, \mathbf{M}, \mathbf{I F}$, and HEF. Let ${ }_{0} \mathbf{x}, 0 \mathbf{y} \in \mathbf{X}$ satisfy ${ }_{0} \mathbf{x} \succ{ }_{0} \mathbf{y}$, and let $\underline{y}, \bar{y} \in Y$ satisfy $\underline{y} \leq x_{t}, y_{t} \leq \bar{y}$ for all $t \in \mathbb{Z}_{+}$. For any $T>0$ with $x_{T-1}>\underline{y}$, Proposition 4 implies that $\left(x_{T-1}\right.$, con $\left.\underline{y}\right) \succ$ con $y$ would contradict RC and HEF. Since $x_{T-1} \geq y$, it follows from $\mathbf{O}$ and $\mathbf{M}$ that $\left(x_{T-1}, \operatorname{con} \underline{y}\right) \sim \operatorname{con} \underline{y}$ for all $T>0$. By IF and the above argument,

$$
\left(T-2 \mathbf{x}_{T-1}, \operatorname{con} \mathrm{Y}\right)=\left(x_{T-2}, x_{T-1}, \operatorname{con} \mathrm{Y}\right) \sim\left(x_{T-2}, \operatorname{con} \mathrm{Y}\right) \sim \operatorname{con} \mathrm{Y}
$$

for all $T>1$. By invoking $\mathbf{O}$ and applying IF and the above argument repeatedly, $\left({ }_{0} \mathbf{x}_{T-1}, \operatorname{con} \underline{y}\right) \sim \operatorname{con} \underline{y}$ for all $T>0$. Likewise, $\left({ }_{0} \mathbf{y}_{T-1}, \operatorname{con} \underline{y}\right) \sim \operatorname{con} \underline{y}$ for all $T>0$.

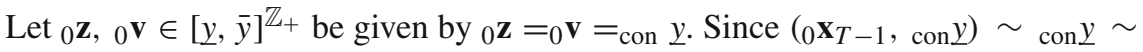
$\left({ }_{0} \mathbf{y}_{T-1}\right.$, con $\left.\underline{y}\right)$ for all $T>0$, we have by $\mathbf{O}$ that $\left({ }_{0} \mathbf{x}_{T-1},{ }_{T} \mathbf{z}\right) \sim\left({ }_{0} \mathbf{y}_{T-1}, T_{T} \mathbf{v}\right)$ for all $T>0$. This contradicts DP.

The following result is useful for the proof of Proposition 6.

Lemma 2 Assume that the SWR $\succsim$ satisfies conditions $\mathbf{O}, \mathbf{R C}, \mathbf{I F}, \mathbf{M}, \mathbf{R D}$, and $\mathbf{H E F}$. Then, for all ${ }_{0} \mathbf{x} \in \mathbf{X}$ and $T \in \mathbb{Z}_{+}, T \mathbf{x} \precsim T+1 \mathbf{x}$.

Proof Assume that the SWR $\succsim$ satisfies conditions O, RC, IF, M, RD, and HEF. By the interpretation of ${ }_{T} \mathbf{x}$, it is sufficient to show that ${ }_{0} \mathbf{x} \precsim{ }_{1} \mathbf{x}$. Suppose on the contrary that ${ }_{0} \mathbf{x} \succ{ }_{1} \mathbf{x}$. By Lemma 1 , there exist $z^{0}, z^{1} \in Y$ such that ${ }_{\text {con }} z^{0} \sim{ }_{0} \mathbf{x}$ and ${ }_{\operatorname{con}} z^{1} \sim{ }_{1} \mathbf{x}$, where by $\mathbf{O}, \mathbf{M}$, and ${ }_{0} \mathbf{x} \succ{ }_{1} \mathbf{x}$, it follows that $z^{0}>z^{1}$. Furthermore, since ${ }_{1} \mathbf{x} \sim{ }_{\text {con }} z^{1}$, it follows by $\mathbf{I F}$ that $\left(x_{0},{ }_{1} \mathbf{x}\right) \sim\left(x_{0}\right.$, con $\left.^{1}\right)$. Hence, ${ }_{0} \mathbf{x} \sim\left(x_{0}\right.$, con $\left.^{1}\right)$. 
If $x_{0} \leq z^{0}$, then,

$$
\begin{aligned}
0 \mathbf{x} & \sim\left(x_{0}, \operatorname{con} z^{1}\right) \prec\left(x_{0}, \operatorname{con} z^{0}\right) & & \text { by (4) and condition IF since } z^{1}<z^{0} \\
& \precsim\left(z^{0}, \operatorname{con} z^{0}\right)=\operatorname{con}^{0} \sim{ }_{0} \mathbf{x} & & \text { by conditions } \mathbf{O} \text { and } \mathbf{M} \text { since } x_{0} \leq z^{0} .
\end{aligned}
$$

This contradicts condition $\mathbf{O}$, ruling out this case.

If $x_{0}>z^{0}$, then, by selecting some $v \in\left(z^{1}, z^{0}\right)$,

$$
\begin{aligned}
& { }_{0} \mathbf{x} \sim\left(x_{0}, \operatorname{con} z^{1}\right) \precsim\left(z^{0}, \operatorname{con} v\right) \text { by conditions } \mathbf{O} \text { and } \mathbf{H E F} \\
& \text { since } x_{0}>z^{0}>v>z^{1} \\
& \prec\left(z^{0},{ }_{\operatorname{con}} z^{0}\right) \sim{ }_{0} \mathbf{x} \quad \text { by (4) and condition IF since } v<z^{0} \text {. }
\end{aligned}
$$

This contradicts condition $\mathbf{O}$, ruling out also this case.

Proof of Proposition 6 Part I: (1) implies (2). Assume that the SWR $\succsim$ satisfies conditions O, RC, IF, M, RD, and HEF. By Proposition 2, the SWR $\succsim$ is represented by a monotone SWF $W: \mathbf{X} \rightarrow \mathbb{R}$ satisfying, for some $U \in \mathcal{U}_{I}$ and $V \in \mathcal{V}(U), W\left({ }_{0} \mathbf{x}\right)=$ $V\left(U\left(x_{0}\right), W\left({ }_{1} \mathbf{x}\right)\right)$ for all ${ }_{0} \mathbf{x} \in \mathbf{X}$ and $W\left({ }_{\operatorname{con}} z\right)=U(z)$ for all $z \in Y$. It remains to be shown that $V(u, w)=w$ for $u>w$, implying that $V$ satisfies $\left(\mathrm{V} .3^{\prime}\right)$ and, thus, $V \in \mathcal{V}_{S}(U)$.

Since $V(u, w)$ is non-decreasing in $u$ for given $w \in U(Y)$ and $V(u, w)=w$ for $u=w$, suppose that $V(u, w)>w$ for some $u, w \in U(Y)$ with $u>w$. Since $U \in \mathcal{U}_{I}$, the properties of $W$ imply that there exist $x, z \in Y$ with $x>z$ such that

$$
\begin{aligned}
W(x, \operatorname{con} z) & =V(U(x), W(\operatorname{con} z))=V(U(x), U(z)) \\
& =V(u, w)>w=U(z)=W(\operatorname{con} z) .
\end{aligned}
$$

Since the SWR $\succsim$ is represented by the SWF $W$, it follows that $(x, \operatorname{con} z) \succ \operatorname{con} z$. This contradicts Lemma 2.

Part II: (2) implies (1). Assume that the monotone mapping $W: \mathbf{X} \rightarrow \mathbb{R}$ is an SWF and satisfies, for some $U \in \mathcal{U}_{I}$ and $V \in \mathcal{V}_{S}(U), W\left({ }_{0} \mathbf{x}\right)=V\left(U\left(x_{0}\right), W\left({ }_{1} \mathbf{x}\right)\right)$ for all ${ }_{0} \mathbf{X} \in \mathbf{X}$ and $W(\operatorname{con} z)=U(z)$ for all $z \in Y$. By Proposition 2 , it remains to be shown that the SWR $\succsim$, represented by the SWF $W$, satisfies HEF. We now provide this argument.

Let $x, y, z, v \in Y$ satisfy $x>y>v>z$. We have to show that $(x, \operatorname{con} z) \nsucc$ $(y, \operatorname{con} v)$, or equivalently, $W(x, \operatorname{con} z) \leq W(y, \operatorname{con} v)$. By the properties of $W$,

$$
\begin{aligned}
W(x, \operatorname{con} z) & =V(U(x), W(\operatorname{con} z))=V(U(x), U(z))=U(z)<U(v) \\
& =V(U(y), U(v))=V(U(y), W(\operatorname{con} v))=W(y, \operatorname{con} v)
\end{aligned}
$$

since $x>y>v>z, U \in \mathcal{U}_{I}$, and $V \in \mathcal{V}_{S}(U)$.

Proof of Proposition 7 Fix $U \in \mathcal{U}_{I}$ and $V \in \mathcal{V}_{S}(U)$. The proof has two parts.

Part I: $\lim _{T \rightarrow \infty} W\left({ }_{T} \mathbf{x}\right)=\liminf _{t \rightarrow \infty} U\left(x_{t}\right)$. Assume that the monotone mapping $W: \mathbf{X} \rightarrow \mathbb{R}$ satisfies $W\left({ }_{0} \mathbf{x}\right)=V\left(U\left(x_{0}\right), W\left({ }_{1} \mathbf{x}\right)\right)$ for all ${ }_{0} \mathbf{x} \in \mathbf{X}$ and $W\left({ }_{\operatorname{con}} z\right)=U(z)$ for all $z \in Y$. Hence, by Proposition 6 , the SWF $W$ represents an SWR $\succsim$ satisfying $\mathbf{O}$, 
RC, M, RD, IF, and HEF. By Lemma 1 , for all ${ }_{0} \mathbf{x} \in \mathbf{X}$, there exists $z \in Y$ such that ${ }_{\operatorname{con}} z \sim{ }_{0} \mathbf{x}$. By Lemma $2, W\left({ }_{t} \mathbf{x}\right)$ is non-decreasing in $t$.

Step 1: $\left.\lim _{t \rightarrow \infty} W{ }_{t} \mathbf{x}\right)$ exists. Suppose $\left.W{ }_{\tau} \mathbf{x}\right)>\lim \sup _{t \rightarrow \infty} U\left(x_{t}\right)$ for some $\tau \in$ $\mathbb{Z}_{+}$. By the premise and the fact that $U \in \mathcal{U}_{I}$, there exists $z \in Y$ satisfying

$$
W\left({ }_{\tau} \mathbf{x}\right) \geq U(z)>\lim \sup _{t \rightarrow \infty} U\left(x_{t}\right)
$$

and $T \geq \tau$ such that $z>v:=\sup _{t \geq T} x_{t}$. By $\mathbf{R D}, \mathbf{O}$, and $\mathbf{M}, \operatorname{con} z \succ(v, \operatorname{con} z) \succsim{ }_{T} \mathbf{x}$, and hence, by $\mathbf{O}$, con $z_{\succ} \mathbf{x}$. However, since $W\left({ }_{t} \mathbf{x}\right)$ is non-decreasing in $t, W\left({ }_{T} \mathbf{x}\right) \geq$ $W\left({ }_{\tau} \mathbf{x}\right) \geq U(z)$. This contradicts that $W$ is an SWF. Hence, $W\left({ }_{t} \mathbf{x}\right)$ is bounded above by $\lim \sup _{t \rightarrow \infty} U\left(x_{t}\right)$, and the result follows since $W\left({ }_{t} \mathbf{x}\right)$ is non-decreasing in $t$.

Step 2: $\lim _{t \rightarrow \infty} W\left(_{t} \mathbf{x}\right) \geq \liminf _{t \rightarrow \infty} U\left(x_{t}\right)$. Suppose

$$
\lim _{t \rightarrow \infty} W\left({ }_{t} \mathbf{x}\right)<\liminf _{t \rightarrow \infty} U\left(x_{t}\right)
$$

By the premise and the fact that $U \in \mathcal{U}_{I}$, there exists $z \in Y$ satisfying

$$
\lim _{t \rightarrow \infty} W\left({ }_{t} \mathbf{x}\right) \leq U(z)<\liminf _{t \rightarrow \infty} U\left(x_{t}\right)
$$

and $T \geq 0$ such that $z<v:=\inf _{t \geq T} x_{t}$. By $\mathbf{O}, \mathbf{M}$, and $\mathbf{R D}, \operatorname{con} z \precsim(z, \operatorname{con} v) \prec$ ${ }_{\text {con }} v \precsim{ }_{T} \mathbf{x}$, and hence, by $\mathbf{O}, \operatorname{con} z \prec{ }_{T} \mathbf{x}$. However, since $W\left({ }_{t} \mathbf{x}\right)$ is non-decreasing in $t, W\left({ }_{T} \mathbf{x}\right) \leq \lim _{t \rightarrow \infty} W\left({ }_{t} \mathbf{x}\right) \leq U(z)$. This contradicts that $W$ is an SWF.

Step 3: $\lim _{t \rightarrow \infty} W\left({ }_{t} \mathbf{x}\right) \leq \liminf _{t \rightarrow \infty} U\left(x_{t}\right)$. Suppose

$$
\lim _{t \rightarrow \infty} W\left({ }_{t} \mathbf{x}\right)>\liminf _{t \rightarrow \infty} U\left(x_{t}\right)
$$

By Lemma 1, there exists, for all $t \in \mathbb{Z}_{+}, z^{t} \in Y$ such that ${ }_{\operatorname{con}} z^{t} \sim{ }_{t} \mathbf{x}$. Since $U \in \mathcal{U}_{I}, z \in Y$ defined by $z:=\lim _{t \rightarrow \infty} z^{t}$ satisfies $U(z)=\lim _{t \rightarrow \infty} W\left({ }_{t} \mathbf{x}\right)$. By the premise and the fact that $U \in \mathcal{U}_{I}$, there exists $x \in Y$ satisfying

$$
\liminf _{t \rightarrow \infty} U\left(x_{t}\right)<U(x)<U(z)
$$

and a subsequence $\left(x_{t_{\tau}}, z^{t_{\tau}}\right)_{\tau \in \mathbb{Z}_{+}}$such that, for all $\tau \in \mathbb{Z}_{+}, x_{t_{\tau}} \leq x<z^{t_{\tau}}$. Then

$$
{ }_{\operatorname{con}} z^{t_{\tau}} \sim{ }_{t_{\tau}} \mathbf{x}=\left(x_{t_{\tau}}, t_{\tau}+1 \mathbf{x}\right) \precsim\left(x, \operatorname{con} z^{t_{\tau+1}}\right) \precsim(x, \operatorname{con} z)
$$

since $z^{t}$ is non-decreasing in $t$. By $\mathbf{O}, \mathbf{R C}$, and the definition of $z, \operatorname{con} z \precsim(x, \operatorname{con} z)$. Since $x<z$, this contradicts RD.

Part II: Existence. Let ${ }_{0} \mathbf{x} \in \mathbf{X}$. This implies that there exist $y, \bar{y} \in Y$ such that, for all $t \in \mathbb{Z}_{+}, \underline{y} \leq x_{t} \leq \bar{y}$. For each $T \in \mathbb{Z}_{+}$, consider $\{w(t, T)\}_{t=0}^{T}$ determined by (1).

Step 1: $w(t, T)$ is non-increasing in $T$ for given $t \leq T$. Given $T \in \mathbb{Z}_{+}$,

$$
\begin{aligned}
w(T, T+1) & =V\left(U\left(x_{T}\right), w(T+1, T+1)\right) \\
& \leq w(T+1, T+1)=\liminf _{t \rightarrow \infty} U\left(x_{t}\right)=w(T, T)
\end{aligned}
$$


by (1) and (V.3'). Thus, applying (V.2), we have

$$
\begin{aligned}
w(T-1, T+1) & =V\left(U\left(x_{T-1}\right), w(T, T+1)\right) \\
& \leq V\left(U\left(x_{T-1}\right), w(T, T)\right)=w(T-1, T) .
\end{aligned}
$$

Using (V.2) repeatedly, we obtain

$$
w(t, T+1) \leq w(t, T) \text { for all } t \in\{0, \ldots, T-1\}
$$

which establishes that $w(t, T)$ is non-increasing in $T$ for given $t \leq T$.

Step 2: $w(t, T)$ is bounded below by $U(y)$. By (1), (V.1), (V.2), and (V.3'), $w(T, T)=\liminf _{t \rightarrow \infty} U\left(x_{t}\right) \geq U(y)$, and for all $t \in\{0, \ldots, T-1\}$,

$w(t+1, T) \geq U(\underline{y}) \Rightarrow w(t, T)=V\left(U\left(x_{t}\right), w(t+1, T)\right) \geq V(U(\underline{y}), U(\underline{y}))=U(\underline{y})$.

Hence, it follows by induction that $w(t, T)$ is bounded below by $U(\underline{y})$.

Step 3: Definition and properties of $W_{\sigma}$. By steps 1 and $2, \lim _{T \rightarrow \infty} w(t, T)$ exists for all $t \in \mathbb{Z}_{+}$. Define the mapping $W_{\sigma}: \mathbf{X} \rightarrow \mathbb{R}$ by $(\mathrm{W})$. We have that $W_{\sigma}$ is monotone by (1), (V.1), and (V.2). As $w(0, T)=V\left(U\left(x_{0}\right), w(1, T)\right)$ and $V$ satisfies (V.0), we have that $W_{\sigma}\left({ }_{0} \mathbf{x}\right)=V\left(U\left(x_{0}\right), W_{\sigma}\left({ }_{1} \mathbf{x}\right)\right)$. Finally, if ${ }_{0} \mathbf{x}={ }_{\operatorname{con}} z$ for some $z \in Y$, then it follows from (1) and (V.3') that $w(t, T)=U(z)$ for all $T \in \mathbb{Z}_{+}$and $t \in\{0, \ldots, T\}$, implying that $W_{\sigma}\left({ }_{0} \mathbf{x}\right)=U(z)$.

Proof of Proposition 8 Suppose there exists a monotone mapping $W: \mathbf{X} \rightarrow \mathbb{R}$ satisfying $W\left(_{0} \mathbf{y}\right)=V\left(U\left(y_{0}\right), W\left({ }_{1} \mathbf{y}\right)\right)$ for all ${ }_{0} \mathbf{y} \in \mathbf{X}$ and $W\left({ }_{c o n} z\right)=U(z)$ for all $z \in Y$ such that $W\left(_{0} \mathbf{x}\right) \neq W_{\sigma}\left({ }_{0} \mathbf{x}\right)$. Since $V$ satisfies the property of weak time perspective, there is a continuous increasing transformation $g: \mathbb{R} \rightarrow \mathbb{R}$ such that $\left|g\left(W\left(_{0} \mathbf{x}\right)\right)-g\left(W_{\sigma}\left({ }_{0} \mathbf{x}\right)\right)\right|=\epsilon>0$, and furthermore, $\left.\mid g\left(W{ }_{t} \mathbf{x}\right)\right)-g\left(W_{\sigma}\left({ }_{t} \mathbf{x}\right)\right) \mid=$ $\left.\left|g\left(V\left(U\left(x_{t}\right), W\left({ }_{t+1} \mathbf{x}\right)\right)\right)-g\left(V\left(U\left(x_{t}\right), W_{\sigma}\left({ }_{t+1} \mathbf{x}\right)\right)\right)\right| \leq \mid g\left(W_{t+1} \mathbf{x}\right)\right)-g\left(W_{\sigma}\left(t_{t+1} \mathbf{x}\right)\right) \mid$ for all $t \in \mathbb{Z}_{+}$. It now follows, by induction, that

$$
\left|g\left(W\left({ }_{T} \mathbf{x}\right)\right)-g\left(W_{\sigma}\left({ }_{T} \mathbf{x}\right)\right)\right| \geq \epsilon>0
$$

for all $T \in \mathbb{Z}_{+}$. However this contradicts that, for all $T \in \mathbb{Z}_{+}$,

$$
\lim _{T \rightarrow \infty} W\left({ }_{T} \mathbf{x}\right)=\liminf _{t \rightarrow \infty} U\left(x_{t}\right)=\lim _{T \rightarrow \infty} W_{\sigma}(\mathbf{x})
$$

by Proposition 7, since $g$ is a continuous increasing transformation.

For the proofs of the results of Sect. 4 , the following notation is useful, where ${ }_{0} \mathbf{z}=\left(z_{0},{ }_{1} \mathbf{z}\right)=\left(z_{0}, z_{1},{ }_{2} \mathbf{z}\right) \in \mathbf{X}$ is a fixed but arbitrary reference stream:

$$
\begin{array}{ll}
x_{0} \succsim_{0}^{\mathbf{z}} y_{0} & \text { means }\left(x_{0},{ }_{1} \mathbf{z}\right) \succsim\left(y_{0},{ }_{1} \mathbf{z}\right) \\
{ }_{1} \mathbf{x}{ }_{1} \succsim^{\mathbf{z}}{ }_{1} \mathbf{y} & \text { means }\left(z_{0},{ }_{1} \mathbf{x}\right) \succsim\left(z_{0}, 1 \mathbf{y}\right) \\
\left(x_{0}, x_{1}\right)_{0} \succsim_{1}^{\mathbf{z}}\left(y_{0}, y_{1}\right) & \text { means }\left(x_{0}, x_{1},{ }_{2} \mathbf{z}\right) \succsim\left(y_{0}, y_{1},{ }_{2} \mathbf{z}\right) \\
{ }_{2} \mathbf{x}{ }_{2} \succsim^{\mathbf{z}}{ }_{2} \mathbf{y} & \text { means }\left(z_{0}, z_{1},{ }_{2} \mathbf{x}\right) \succsim\left(z_{0}, z_{1},{ }_{2} \mathbf{y}\right) \\
x_{1} \succsim_{1}^{\mathbf{z}} y_{1} & \text { means }\left(z_{0}, x_{1},{ }_{2} \mathbf{z}\right) \succsim\left(z_{0}, y_{1},{ }_{2} \mathbf{z}\right) .
\end{array}
$$


Say that $\succsim_{0}^{\mathbf{z}}$ is independent of ${ }_{0} \mathbf{z}$ if, for all ${ }_{0} \mathbf{x},{ }_{0} \mathbf{y},{ }_{0} \mathbf{z},{ }_{0} \mathbf{v} \in \mathbf{X}, x_{0} \succsim_{0}^{\mathbf{z}} y_{0}$ if and only if $x_{0} \succsim_{0}^{\mathbf{v}} y_{0}$, and likewise for ${ }_{1} \succsim^{\mathbf{z}}, 0 \succsim_{1}^{\mathbf{z}},{ }_{2} \succsim^{\mathbf{z}}$, and $\succsim_{1}^{\mathbf{z}}$. In this notation and terminology, condition IF implies that ${ }_{1} \succsim^{\mathbf{z}}$ is independent of $0 \mathbf{z}$, while condition IP states that $0 \succsim_{1}^{\mathbf{z}}$ is independent of ${ }_{0} \mathbf{z}$. The following result due to Gorman (1968b) indicates that imposing condition IP is consequential.

Lemma 3 Assume that the SWR $\succsim$ satisfies conditions IF and IP. Then $\succsim_{0}^{\mathbf{z}},{ }_{1} \succsim^{\mathbf{z}},{ }_{0} \succsim_{1}^{\mathbf{z}},{ }_{2} \succsim^{\mathbf{z}}$, and $\succsim_{1}^{\mathbf{z}}$ are independent of ${ }_{0} \mathbf{z}$.

Proof Assume that the SWR $\succsim$ satisfies conditions IF and IP. By repeated application of IF , ${ }_{1} \succsim^{\mathbf{z}}$ and ${ }_{2} \succsim^{\mathbf{z}}$ are independent of ${ }_{0} \mathbf{z}$, while IP states that ${ }_{0} \succsim_{1}^{\mathbf{z}}$ is independent of ${ }_{0} \mathbf{z}$. By IF,$\left(x_{1}, 2 \mathbf{z}\right) \succsim\left(y_{1}, 2 \mathbf{z}\right)$ is equivalent to $\left(z_{0}, x_{1},{ }_{2} \mathbf{z}\right) \succsim\left(z_{0}, y_{1}, 2_{2} \mathbf{z}\right)$, which, by IP, is equivalent to $\left(z_{0}, x_{1}, 2 \mathbf{v}\right) \succsim\left(z_{0}, y_{1}, 2 \mathbf{v}\right)$, which in turn, by IF, is equivalent to $\left(x_{1}, 2 \mathbf{v}\right) \succsim\left(y_{1}, 2 \mathbf{v}\right)$, which finally, by $\mathbf{I F}$, is equivalent to $\left(v_{0}, x_{1}, 2 \mathbf{v}\right) \succsim$ $\left(v_{0}, y_{1}, 2 \mathbf{v}\right)$, where $0 \mathbf{v} \in \mathbf{X}$ is some arbitrary stream. Hence, $\succsim_{0}^{z}$ and $\succsim_{1}^{z}$ are independent of $0 \mathbf{z}$.

Proof of Theorem 2 Part I: This part is proved in three steps.

Step 1: By Lemma 3, IF and IP imply that $\succsim_{0}^{\mathbf{z}}$ is independent of ${ }_{0} \mathbf{z}$.

Step 2: By condition WS, there exist ${ }_{0} \mathbf{x},{ }_{0} \mathbf{y},{ }_{0} \mathbf{z} \in \mathbf{X}$ such that $x_{0} \succ_{0}^{\mathbf{z}} y_{0}$. This rules out that $x_{0}=y_{0}$, and by $\mathbf{M}, x_{0}<y_{0}$ would lead to a contradiction. Hence, $x_{0}>y_{0}$. Since $\succsim_{0}^{\mathbf{z}}$ is independent of ${ }_{0} \mathbf{z}$, this implies $\mathbf{R S}$.

Step 3: By Proposition 4, there is no SwR $\succsim$ satisfying RC, RS, and HEF.

Part II: To establish this part, consider dropping a single condition.

Dropping IP. Existence follows from Theorem 1 since RD implies WS.

Dropping HEF. Existence follows from Propositions 9 and 10.

Dropping WS. All the remaining conditions are satisfied by the SWF $\succsim$ being represented by the mapping $W: \mathbf{X} \rightarrow \mathbb{R}$ defined by $W\left(_{0} \mathbf{x}\right):=\liminf _{t \rightarrow \infty} x_{t}$.

Dropping M. All the remaining conditions are satisfied by the SWF $\succsim$ being represented by the mapping $W: \mathbf{X} \rightarrow \mathbb{R}$ defined by $W\left({ }_{0} \mathbf{x}\right):=-x_{0}+\liminf _{t \rightarrow \infty} x_{t}$.

Dropping IF. All the remaining conditions are satisfied by the SWF $\succsim$ being represented by the mapping $W: \mathbf{X} \rightarrow \mathbb{R}$ defined by $W\left(_{0} \mathbf{x}\right):=\min \left\{x_{0}, x_{1}\right\}$.

Dropping RC. Existence follows from Proposition 13 since SP implies $\mathbf{M}$ and WS.

Proof of Proposition 9 The proof is based on standard results for additively separable representations (Debreu 1960; Gorman 1968a; Koopmans 1986a), and is available at http://folk.uio.no/gasheim/srswfs2.pdf

Proof of Proposition 10 Available at http://folk.uio.no/gasheim/srswfs2.pdf

Proof of Proposition 11 Assume that the SwR $\succsim$ satisfies conditions $\mathbf{O}, \mathbf{R C}, \mathbf{I F}, \mathbf{M}$, IP, and NDF. By Proposition 1, O, IF, and NDF imply WS. Hence, by Propositions 9 and 10 , the SWR $\succsim$ is represented by $W_{\delta}: \mathbf{X} \rightarrow \mathbb{R}$ defined by, for each ${ }_{0} \mathbf{X} \in \mathbf{X}$, 


$$
W_{\delta}\left({ }_{0} \mathbf{x}\right)=(1-\delta) \sum_{t=0}^{\infty} \delta^{t} U\left(x_{t}\right)
$$

for some $U \in \mathcal{U}$ and $\delta \in(0,1)$. This implies DP, thus contradicting NDP.

The proof of Proposition 12 needs some preliminaries. A sustainable recursive SWF $W: \mathbf{X} \rightarrow \mathbb{R}$ is given, with $W\left({ }_{0} x\right)=V\left(U\left(x_{0}\right), W\left({ }_{1} x\right)\right)$ for all ${ }_{0} x \in \mathbf{X}$ and $W(\operatorname{con} z)=U(z)$ for all $z \in Y$. A utility stream ${ }_{0} \mathbf{u}$ is associated with a feasible path ${ }_{0} \mathbf{k}$ from $k \in[0, \bar{k}]$ if $u_{t}=U\left(x\left(f\left(k_{t}\right)-k_{t+1}, k_{t}\right)\right)$ for $t \geq 0$. Write $u^{*} \equiv U\left(x^{*}\right)=$ $U\left(x\left(c^{*}, k^{*}\right)\right)=U\left(x\left(f\left(k^{*}\right)-k^{*}, k^{*}\right)\right)$.

Write $S:=\left\{(c, k) \in[0, \bar{k}] \mid x(c, k)=x^{*}\right\}$. Since $S \neq \emptyset$, we can define

$$
I=\{k \in(0, \bar{k}] \mid \text { there is some } c \geq 0 \text { satisfying }(c, k) \in S\}
$$

Note that $k^{*} \in I$. Let $k \in I$; then there is $c \geq 0$ such that $x(c, k)=x^{*}$. Now, let $k^{\prime} \in I$ satisfy $k^{\prime}>k$. Then $x\left(c, k^{\prime}\right) \geq x(c, k)=x^{*}$, while $x\left(0, k^{\prime}\right) \leq x(0, \bar{k})=$ $x(f(\bar{k})-\bar{k}, \bar{k}) \leq x^{*}$. Thus, by continuity of $x$, there is some $c^{\prime} \geq 0$, such that $x\left(c^{\prime}, k^{\prime}\right)=x^{*}$. This shows that $I$ is a sub-interval of $(0, \bar{k}]$, containing $\left[k^{*}, \bar{k}\right]$.

Define, for each $k \in I$, the set $\phi(k)=\{c \geq 0 \mid(c, k) \in S\}$. By definition of $I, \phi(k)$ is non-empty for each $k \in I$. Since $k \in I$ implies $k>0, \phi(k)$ is a singleton by property (3)(ii) of the function $x$. Thus, $\phi$ is a function from $I$ to $\mathbb{R}_{+}$, and by definition, $x(\phi(k), k)=x^{*}$ for all $k \in I$, so $c^{*}=\phi\left(k^{*}\right)$. By property (3)(ii), $\phi$ is non-increasing on $I$.

Lemma 4 For every $k \in\left[k^{*}, \bar{k}\right]$, there exists a feasible resource path, ${ }_{0} \hat{\mathbf{k}}$, from $k$ where the associated well-being stream, ${ }_{0} \hat{\mathbf{x}}$, satisfies $W\left({ }_{t} \hat{\mathbf{x}}\right)=W\left({ }_{\operatorname{con}} x^{*}\right)$ for $t \geq 0$.

Proof Let $k \in\left[k^{*}, \bar{k}\right]$, and consider the resource path ${ }_{0} \hat{\mathbf{k}}$ defined by

$$
k_{0}=k, \quad k_{t+1}=f\left(k_{t}\right)-\phi\left(k_{t}\right) \text { for } t \geq 0 .
$$

Note that, if $k_{t} \in\left[k^{*}, \bar{k}\right]$, then

$$
\bar{k} \geq f(\bar{k}) \geq f\left(k_{t}\right)-\phi\left(k_{t}\right) \geq f\left(k_{t}\right)-\phi\left(k^{*}\right)=f\left(k_{t}\right)-\left[f\left(k^{*}\right)-k^{*}\right] \geq k^{*} .
$$

Hence, $k_{t+1} \in\left[k^{*}, \bar{k}\right]$ and, by induction, $k_{t} \in\left[k^{*}, \bar{k}\right]$ for $t \geq 0$. This shows that ${ }_{0} \hat{\mathbf{k}}$ is feasible from $k \in\left[k^{*}, \bar{k}\right]$. By the definition of $\phi(\cdot), x_{t}=x\left(\phi\left(k_{t}\right), k_{t}\right)=x^{*}$ for $t \geq 0$.

Lemma 5 Let ${ }_{0} \hat{\mathbf{k}}$ be a feasible resource path from $k \in[0, \bar{k}]$ with associated utility stream, ${ }_{0} \mathbf{u}$. Given any $\varepsilon>0$, there is some $T \geq 0$ such that $u_{T}<u^{*}+\varepsilon$.

Proof Suppose, on the contrary, there is some $\varepsilon>0$, such that $u_{t} \geq u^{*}+\varepsilon$ for all $t \geq 0$. By continuity of $U$, there is $\delta>0$, such that whenever $x \in Y$ and $\left|x-x^{*}\right|<\delta$, we have $\left|U(x)-U\left(x^{*}\right)\right|<\varepsilon$. Thus, we must have $\left|x_{t}-x^{*}\right| \geq \delta$ for all $t \geq 0$. Further, since $U$ is an increasing function, we must have $x_{t} \geq x^{*}+\delta$ for all $t \geq 0$. This implies

$$
x\left(f\left(k_{t}\right)-k_{t+1}, k_{t}\right)=x\left(c_{t}, k_{t}\right)>x^{*} \text { for all } t \geq 0 .
$$


Since $x\left(f\left(k_{t}\right)-k_{t}, k_{t}\right) \leq x^{*}$, property (3)(ii) implies that $k_{t+1}<k_{t}$ for all $t \geq 0$. Thus, ${ }_{0} \mathbf{k}$ must converge to some $\kappa \in[0, \bar{k}]$. The continuity of $f$ and $x$ then imply that $x(f(\kappa)-\kappa, \kappa) \geq x^{*}+\delta$, and this contradicts the definition of $x^{*}$.

Lemma 6 Let ${ }_{0} \hat{\mathbf{k}}$ be a feasible resource path from $k \in[0, \bar{k}]$ with associated wellbeing stream, ${ }_{0} \hat{\mathbf{x}}$. Then, we have $W\left({ }_{0} \hat{\mathbf{x}}\right) \leq W\left({ }_{\operatorname{con}} x^{*}\right)$.

Proof Suppose, by way of contradiction, that there exist $k \in[0, \bar{k}]$ and a feasible resource path, ${ }_{0} \hat{\mathbf{k}}$, from $k$ where the associated well-being stream, ${ }_{0} \hat{\mathbf{x}}$, satisfies $W\left({ }_{0} \hat{\mathbf{x}}\right)>$ $W\left(\operatorname{con}^{*}\right)=U\left(x^{*}\right)=u^{*}$. Denote by ${ }_{0} \hat{\mathbf{u}}$ the associated utility stream (i.e., $\hat{u}_{t}=U\left(\hat{x}_{t}\right)$ for $t \geq 0$ ). Since $\left.W{ }_{t} \hat{\mathbf{x}}\right)$ is non-decreasing in $t$, and is bounded above by $U(x(\bar{k}, \bar{k}))$ (by the properties of a sustainable recursive SWF), it converges to some $\omega \leq U(x(\bar{k}, \bar{k}))$. Hence, $\left.\omega \geq W{ }_{0} \hat{\mathbf{x}}\right)>u^{*}$. Since the aggregator function $V$ satisfies $\left(\bar{V} .3^{\prime}\right)$, we must have $V\left(u^{*}, \omega\right)<V(\omega, \omega)=\omega$. Using the continuity of $V$, we can find $\varepsilon>0$ such that

$$
V\left(u^{*}+\varepsilon, \omega\right)<V(\omega, \omega)=\omega
$$

Write $\theta:=\omega-V\left(u^{*}+\varepsilon, \omega\right)$. By (5), $\theta>0$.

Choose $T \in Z_{+}$large enough so that for all $t \geq T, W\left({ }_{t} \hat{\mathbf{x}}\right) \geq \omega-(\theta / 2)$. By Lemma $5, \hat{u}_{t}<u^{*}+\varepsilon$ for some $t \geq T$. Let $\tau$ be the first period $(\geq T$ ) for which $\hat{u}_{t}<u^{*}+\varepsilon$. Then,

$$
\begin{aligned}
\omega-\frac{\theta}{2} & \leq W\left({ }_{\tau} \hat{\mathbf{x}}\right)=V\left(u_{\tau}, W\left({ }_{\tau+1} \hat{\mathbf{x}}\right)\right) \\
& \leq V\left(u^{*}+\varepsilon, W\left({ }_{\tau+1} \hat{\mathbf{x}}\right)\right) \leq V\left(u^{*}+\varepsilon, \omega\right)=\omega-\theta<\omega-\frac{\theta}{2},
\end{aligned}
$$

which is a contradiction.

Lemma 7 If a feasible resource path, ${ }_{0} \hat{\mathbf{k}}$, from $k \in[0, \bar{k}]$ has an associated well-being stream, ${ }_{0} \hat{\mathbf{x}}$, which satisfies $W\left({ }_{0} \hat{\mathbf{x}}\right)=W\left({ }_{\operatorname{con}} x^{*}\right)$, then $(i) \hat{x}_{t} \geq x^{*}$ for all $t \geq 0$; and (ii) $\hat{k}_{t} \geq k^{*}$ for all $t \geq 0$.

Proof Assume that a feasible resource path, ${ }_{0} \hat{\mathbf{k}}$, from $k \in[0, \bar{k}]$ has an associated wellbeing stream, ${ }_{0} \hat{\mathbf{x}}$, which satisfies $W\left({ }_{0} \hat{\mathbf{x}}\right)=W\left({ }_{\operatorname{con}} x^{*}\right)$. Since $W\left({ }_{t} \hat{\mathbf{x}}\right)$ is non-decreasing in $t$, it follows from Lemma 6 that $W\left({ }_{t} \hat{\mathbf{x}}\right)=W\left({ }_{\operatorname{con}} x^{*}\right)=u^{*}$ for all $t \geq 0$.

To establish (i), suppose, by way of contradiction, that $\hat{x}_{t}<x^{*}$ for some $t \geq 0$. Then, since $U\left(\hat{x}_{t}\right)<U\left(x^{*}\right)=u^{*},\left(\right.$ V. $\left.3^{\prime}\right)$ implies

$$
u^{*}=W\left({ }_{t} \hat{\mathbf{x}}\right)=V\left(U\left(\hat{x}_{t}\right), u^{*}\right)<V\left(u^{*}, u^{*}\right)=u^{*},
$$

which is a contradiction.

To establish (ii), suppose, on the contrary, that $\hat{k}_{\tau}<k^{*}$ for some $\tau \geq 0$. Then, by the fact that $x(f(k)-k, k)<x^{*}$ if $k \neq k^{*}$, we have $x\left(f\left(\hat{k}_{\tau}\right)-\hat{k}_{\tau}, \hat{k}_{\tau}\right)<x^{*}$, while:

$$
x^{*} \leq \hat{x}_{\tau}=x\left(\hat{c}_{\tau}, \hat{k}_{\tau}\right)=x\left(f\left(\hat{k}_{\tau}\right)-\hat{k}_{\tau+1}, \hat{k}_{\tau}\right)
$$


So, $\hat{k}_{\tau+1}<\hat{k}_{\tau}<k^{*}$, and repeating this step, $\hat{k}_{t+1}<\hat{k}_{t}$ for all $t \geq \tau$. Thus, ${ }_{0} \hat{k}$ must converge to some $\kappa \in[0, \bar{k}]$, with $\kappa \leq \hat{k}_{\tau}<k^{*}$. The continuity of $f$ and $x$ then implies that

$$
x(f(\kappa)-\kappa, \kappa) \geq x^{*}
$$

using (i). But, this contradicts that $x(f(k)-k, k)<x^{*}$ if $k \neq k^{*}$.

Proof of Proposition 12 Lemmas 4 and 6 establish existence of an optimum and that any optimal well-being stream satisfies $W\left({ }_{t} \hat{\mathbf{x}}\right)=W\left({ }_{\operatorname{con}} x^{*}\right)$ for $t \geq 0$. Lemma 7 shows that any optimal resource path ${ }_{0} \hat{\mathbf{k}}$, with associated well-being stream ${ }_{0} \hat{\mathbf{x}}$, satisfies $\hat{x}_{t} \geq x^{*}$ and $\hat{k}_{t} \geq k^{*}$ for $t \geq 0$.

Proof of Proposition 13 Asheim and Tungodden (2004a), Basu and Mitra (2007), and Bossert et al. (2007) define different incomplete leximin and undiscounted utilitarian SWRs, each of which is given an axiomatic characterization. Denote by $\succsim$ one such incomplete SWR. It can be verified that $\succsim$ is reflexive, transitive and satisfies IF, SP, HEF (with $(x, \operatorname{con} z) \precsim(y, \operatorname{con} v)$ if $x>y>v>z$ ), and IP. Completeness (and thereby condition O) can be satisfied by invoking Arrow's (1951) version of Szpilrajn's (1930) extension theorem (see also Svensson 1980).

Since $\succsim$ satisfies conditions SP and HEF (with $(x, \operatorname{con} z) \precsim(y, \operatorname{con} v)$ if $x>y>$ $v>z)$, so will any completion. Since, for all ${ }_{0} \mathbf{x},{ }_{0} \mathbf{y},{ }_{0} \mathbf{z} \in \mathbf{X},\left(x_{0}, x_{1}\right){ }_{0} \succsim_{1}^{\mathbf{z}}\left(y_{0}, y_{1}\right)$ or $\left(x_{0}, x_{1}\right) \quad \precsim_{1}^{\mathbf{z}}\left(y_{0}, y_{1}\right)$, and $\succsim$ satisfies IP, so will any completion. However, special care must be taken to ensure that the completion satisfies IF.

Consider $\mathbf{X}_{0}^{2}=\left\{\left(_{0} \mathbf{x}, 0 \mathbf{y}\right) \in \mathbf{X}^{2} \mid x_{0} \neq y_{0}\right\}$, and invoke Arrow's (1951) version of Szpilrajn's (1930) extension theorem to complete $\succsim$ on this subset of $\mathbf{X}^{2}$. For any $\left({ }_{0} \mathbf{x}, 0 \mathbf{y}\right) \in \mathbf{X}$ with ${ }_{0} \mathbf{x} \neq{ }_{0} \mathbf{y}$, let ${ }_{0} \mathbf{x}$ be at least as good as ${ }_{0} \mathbf{y}$ if and only if ${ }_{T} \mathbf{x}$ is at least as good as ${ }_{T} \mathbf{y}$ according to the completion of $\succsim$ on $\mathbf{X}_{0}^{2}$, where $T:=\min \left\{t \mid x_{t} \neq y_{t}\right\}$. Since $\succsim$ satisfies IF, this construction constitutes a complete sWR satisfying IF.

Open Access This article is distributed under the terms of the Creative Commons Attribution Noncommercial License which permits any noncommercial use, distribution, and reproduction in any medium, provided the original author(s) and source are credited.

\section{References}

Alcantud, J.C.R., García-Sanz, M.D.: Paretian evaluation of infinite utility streams: an egalitarian criterion. Econ Lett 106, 209-211 (2010)

Arrow, K.J.: Social Choice and Individual Values. New York: Wiley (1951)

Asheim, G.B.: Unjust intergenerational allocations. J Econ Theory 54, 350-371 (1991)

Asheim, G.B., Mitra, T.: Sustainability and discounted utilitarianism in models of economic growth. Math Soc Sci 59, 148-169 (2010)

Asheim, G.B., Mitra, T., Tungodden, B: A new equity condition for infinite utility streams and the possibility of being Paretian. In: Roemer, J., Suzumura, K. (eds.) Intergenerational Equity and Sustainability, pp. 55-68. Basingstoke: Palgrave Macmillan (2007)

Asheim, G.B., Tungodden, B.: Resolving distributional conflicts between generations. Econ Theory 24, 221$230(2004 \mathrm{a})$

Asheim, G.B., Tungodden, B.: Do Koopmans' postulates lead to discounted utilitarianism? Discussion Paper 32/04, Norwegian School of Economics and Business Administration (2004b) 
Atkinson, A.B.: The strange disappearance of welfare economics. Kyklos 54, 193-206 (2001)

Banerjee, K.: On the equity-efficiency trade off in aggregating infinite utility streams. Econ Lett 93, 6367 (2006)

Basu, K., Mitra, T.: Aggregating infinite utility streams with intergenerational equity: the impossibility of being Paretian. Econometrica 32, 1557-1563 (2003)

Basu, K., Mitra, T.: Utilitarianism for infinite utility streams: a new welfare criterion and its axiomatic characterization. J Econ Theory 133, 350-373 (2007)

Birchenhall, C.R., Grout, P.: On equal plans with an infinite horizon. J Econ Theory 21, 249-264 (1979)

Blackorby, C., Donaldson, D., Weymark, J.A.: Social choice with interpersonal utility comparisons: a diagrammatic introduction. Int Econ Rev 25, 327-356 (1984)

Bleichrodt, H., Rohde, K.I.M., Wakker, P.P.: Koopmans' constant discounting for intertemporal choice: A simplification and a generalization. J Math Psych 52, 341-347 (2008)

Bossert, W., Sprumont, Y., Suzumura, K.: Ordering infinite utility streams. J Econ Theory 135, 579589 (2007)

Burniaux, J.-M., Martins, J.O.: Carbon leakages: A general equilibrium view. Econ Theory (this issue) (2010)

Chichilnisky, G.: An axiomatic approach to sustainable development. Soc Choice Welfare 13, 231257 (1996)

Chichilnisky, G.: Sustainable markets with short sales. Econ Theory (this issue) (2010)

Chichilnisky, G., Heal, G.M., Beltratti, A.: The green golden rule. Econ Lett 49, 175-179 (1995)

Chipman, J.S., Tian, G.: Detrimental externalities, pollution rights, and the "Coase Theorem". Econ Theory (this issue) (2010)

Dasgupta, P.S., Heal, G.M.: The optimal depletion of exhaustible resources. Rev Econ Stud (Symposium) 3-28 (1974)

Dasgupta, P.S., Heal, G.M.: Economic Theory and Exhaustible Resources. Cambridge: Cambridge University Press (1979)

Debreu, G.: Topological methods in cardinal utility theory. In: Arrow, K.J., Karlin, S., Suppes, P. (eds.) Mathematical Methods in the Social Sciences, pp. 16-26. Stanford: Stanford University Press (1960)

Diamond, P.: The evaluation of infinite utility streams. Econometrica 33, 170-177 (1965)

Dutta, P.K., Radner, R.: Capital growth in a global warming model: Will China and India sign a climate treaty? Econ Theory (this issue) (2010)

Figuieres, C., Tidball, M.: Sustainable exploitation of a natural resource: a satisfying use of Chichilnisky's criterion. Econ Theory (this issue) (2010)

Fleurbaey, M., Michel, P.: Transfer principles and inequality aversion, with application to optimal growth. Math Soc Sci 42, 1-11 (2001)

Fleurbaey, M., Michel, P.: Intertemporal equity and the extension of the Ramsey criterion. J Math Econ 39, 777-802 (2003)

Gorman, W.M.: Conditions for additive separability. Econometrica 36, 605-609 (1968a)

Gorman, W.M.: The structure of utility functions. Rev Econ Stud 35, 367-390 (1968b)

Hammond, P.J.: Equity, Arrow's conditions and Rawls' difference principle. Econometrica 44, 793804 (1976)

Hara, C., Shinotsuka, T., Suzumura, K., Xu, Y.: Continuity and egalitarianism in the evaluation of infinite utility streams. Soc Choice Welfare 31, 179-191 (2008)

Heal, G.M.: Valuing the future: Economic theory and sustainability. New York: Columbia University Press (1998)

Heal, G.M.: Intergenerational welfare economics and the environment. In: Mäler, K.-G., Vincent, J. (eds.) Handbook of Environmental Economics, Amsterdam: North-Holland (2005)

Karp, C., Zhang, J.: Taxes versus quantities for a stock pollutant with endogenous abatement costs and asymmetric information. Econ Theory (this issue) (2010)

Koopmans, T.C.: Stationary ordinal utility and impatience. Econometrica 28, 287-309 (1960)

Koopmans, T.C.: Intertemporal distribution and optimal aggregate economic growth. In: Ten Economic Studies in the Tradition of Irving Fisher. New York: Wiley (1967)

Koopmans, T.C.: Representation of preference orderings with independent components of consumption. In: McGuire, C.B., Radner, R. (eds.) Decision and Organization: A Volume in Honor of Jacob Marschak, 2nd edn, pp. 57-78 [previously published in 1972]. Minneapolis: University of Minnesota Press (1986a) 
Koopmans, T.C.: Representation of preference orderings over time. In: McGuire, C.B., Radner, R. (eds.) Decision and Organization: A Volume in Honor of Jacob Marschak, 2nd edn, pp. 57-78 [previously published in 1972]. Minneapolis: University of Minnesota Press (1986b)

Koopmans, T.C., Diamond, P.E., Williamson, R.E.: Stationary utility and time perspective. Econometrica 32, 82-100 (1964)

Krautkraemer, J.A.: Optimal growth, resource amenities and the preservation of natural environments. Rev Econ Stud 52, 153-170 (1985)

Lauwers, L.: Rawlsian equity and generalised utilitarianism with an infinite population. Econ Theory 9, 143150 (1997)

Lauwers, L.: Intertemporal objective functions: Strong Pareto versus anonymity. Math Soc Sci 35, 3755 (1998)

Lauwers, L.: Intergenerational equity, efficiency, and constructibility. Econ Theory (this issue) (2010)

Lecocq, F., Hourcade, J.-C.: Insights from a theoretical analysis of negotiation mandates: Unspoken ethical issues in the climate affair. Econ Theory (this issue) (2010)

Mitra, T.: Stationary Paretian SWF: An Example. Mimeo: Cornell University (2008)

Ostrom, E.: Nested externalities and polycentric institutions: must we wait for global solutions to climate change before taking actions at other scales? Econ Theory (this issue) (2010)

Rawls, J.: A Theory of Justice. Cambridge: Harvard University Press (1971)

Rezai, A., Foley, D.K., Taylor, L.: Global warming and economic externalities. Econ Theory (this issue) (2010)

Solow, R.M.: Intergenerational equity and exhaustible resources. Rev Econ Stud (Symposium), 29-45 (1974)

Svensson, L.-G.: Equity among generations. Econometrica 48, 1251-1256 (1980)

Szpilrajn, E.: Sur l'extension du l'ordre partiel. Fundam Math 16, 386-389 (1930) 